\title{
Abundance difference between components of wide binaries
}

\section{The southern sample ${ }^{\star, \star \star, \star \star \star}$}

\author{
S. Desidera, R. G. Gratton, S. Lucatello, and R. U. Claudi
}

INAF - Osservatorio Astronomico di Padova, Vicolo dell' Osservatorio 5, 5122 Padova, Italy

e-mail: desidera@pd.astro.it

Received 23 January 2006 / Accepted 14 March 2006

ABSTRACT

\begin{abstract}
Aims. We present high-precision iron abundance differences for 33 wide binaries with similar components. They were observed with the FEROS spectrograph at ESO, looking for abundance anomalies due to the ingestion of metal rich material of a planetary origin. Methods. An optimized data analysis technique and the high quality of the spectra allowed us to achieve an error of about 0.02 dex for pairs with small temperaure differences.

Results. We found one case (HIP $64030=$ HD 113984) with a large $(0.27$ dex) abundance difference. The primary of this binary appears to be a blue straggler, and the abundance difference might be due to the peculiar evolution of the star. A few other pairs show small abundance differences $(\leq 0.09 \mathrm{dex})$. In a few cases these differences suggest the ingestion of a small amount of metal rich material, but in others they are likely spurious, because of the large temperature difference, high level of magnetic activity, or different evolutionary phases between the components. Some cases of abundance differences involving pairs with warm $\left(T_{\text {eff }} \geq 6000 \mathrm{~K}\right)$ primaries might be due to the diffusion of heavy elements; dedicated theoretical models for the stellar parameters of the targets would be welcome.

Conclusions. This study confirms our preliminary result based on analysis of 23 pairs (Desidera et al. 2004) that the occurrence of large alterations in stellar abundances caused by the ingestion of metal rich, rocky material is not a common event. For at least $65 \%$ of the pairs with components warmer than $5500 \mathrm{~K}$, the limits on the amount of rocky material accreted by the program stars are comparable to the estimates of rocky material accreted by the Sun during its main-sequence lifetime.
\end{abstract}

Key words. stars: abundances - stars: planetary systems - stars: binaries: visual - techniques: spectroscopic

\section{Introduction}

Wide visual binaries with similar components are ideal targets for high-precision differential abundance measurements. Gratton et al. (2001) and Desidera et al. (2004, hereafter Paper I) showed that errors in estimating the difference of iron content between the two components that are lower than 0.02 dex can be achieved for pairs with temperature differences smaller than 300-400 $\mathrm{K}$ and components with effective temperatures in the range $5300-6300 \mathrm{~K}$. This opens possibilities for a detailed and quantitative study of chemical alterations in the external convective layer, which is caused by the accretion of metal-rich planetary material occurring during the main-sequence lifetime of the stars. In fact, the engulfment of $1 M_{\oplus}$ of iron (about $5 M_{\oplus}$ of meteoritic material) corresponds to an iron abundance difference of about 0.01 dex for a solar type main sequence star.

Accretion phenomena have been suggested to explain the correlation between the presence of giant planets and the high metallicity of their parent stars (Gonzalez 1997). The most recent studies (Santos et al. 2004; Fischer \& Valenti 2005)

* Based on observations collected at the European Southern Observatory, Chile, using FEROS spectrograph (proposals ID: 69.D-0338-70.D-0081).

$\star \star$ Table 3 is only available in electronic form at the CDS via anonymous ftp to cdsarc.u-strasbg.fr $(130.79 .128 .5)$ or via http://cdsweb.u-strasbg. fr/cgi-bin/qcat?J/A+A/454/581

$\star \star \star$ Tables $2,5,6,8-10$ are only available in electronic form at http://www.edpsciences.org conclude that the high metallicity of a planet host is more likely primordial than due to accretion. Nevertheless, the occurrence of planetary pollution has been proposed in some cases (e.g. Israelian et al. 2003; Laws \& Gonzalez 2001).

In Paper I, we studied 23 pairs from the sample of the on-going radial-velocity planet search using SARG at TNG. We did not find any pair with an abundance difference larger than $0.07 \mathrm{dex}$, and most of the pairs have identical iron content within 0.02 dex. However, the relatively small number of targets limits any inferences on the frequency of pairs with enriched components and on the origin of the planet-metallicity connection.

Therefore, we extended our sample by observing a sample of southern visual binaries using the FEROS spectrograph at ESO La Silla. The spectra were analyzed using the same technique as developed in Gratton et al. (2001) and Paper I.

\section{Observations and data reduction}

A sample of 56 wide binaries with similar components (main sequence or slightly evolved stars with a magnitude difference less than 1.0$)^{1}$ were observed with the FEROS spectrograph in two runs (August 2001 and March 2002). Details on the observations and data reduction are found in the companion paper Desidera et al. (2006b, hereafter Paper II), where the study of radial and

1 Two pairs with larger magnitude difference, HIP 76446 and HIP 78024, were also observed and included in this work. 
Table 1. Visual and absolute magnitudes, $B-V$ color, Hipparcos parallaxes, stellar masses (derived iterativey during the abundance analysis using the spectroscopic temperatures and abundances), and projected binary separation for program stars. The formal errors on magnitudes and colors are probably underestimated (see Paper I).

\begin{tabular}{|c|c|c|c|c|c|c|c|c|c|c|c|}
\hline Object & $V_{\mathrm{A}}$ & $V_{\mathrm{B}}$ & $\overline{(B-V)_{\mathrm{A}}}$ & $\overline{\overline{(B-V)_{\mathrm{B}}}}$ & $\begin{array}{c}\pi \\
\text { mas }\end{array}$ & $\overline{\overline{M_{V(\mathrm{~A})}}}$ & $\overline{\bar{M}}$ & $\begin{array}{l}M_{\mathrm{A}} \\
M_{\odot} \\
\end{array}$ & $\begin{array}{l}M_{\mathrm{B}} \\
M_{\odot} \\
\end{array}$ & $\rho$ & $\begin{array}{c}\rho \\
\mathrm{AU} \\
\end{array}$ \\
\hline HIP 3290 & 4 & 8 & 0 & 8 & $.33 \pm$ & \pm & 26 & 1.05 & 0.96 & 6.3 & 645 \\
\hline HIP & & & & 6 & & & & 1.00 & 22 & 5.9 & 0 \\
\hline HIP 2 & & & & & & & & 1.08 & & 5.6 & 152 \\
\hline HIP 3 & & & & 3 & & & & 0. & & 23.1 & 802 \\
\hline HIP 3 & 9 & & & & 1 & 4 & & & & .1 & 0 \\
\hline $4 / 17$ & 9 & 9 & 0 & 0 & 39 & & 0 & 0.97 & 2 & .6 & $1 / 4$ \\
\hline 20 & 8 & 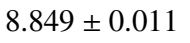 & & 8 & 1 & & 3 & 1. & 9 & 9.6 & 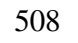 \\
\hline 10 & 6 & 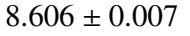 & & 0 & 4 & 5 & 2 & 0.93 & 0.89 & 9 & 19 \\
\hline 80 & $\perp$ & & & 23 & 0 & 3 & 9 & 1. & 1.03 & 9.5 & 542 \\
\hline 30 & 5 & & 8 & 007 & 5 & & 97 & 1.23 & 1.21 & 9.5 & 253 \\
\hline 8 & 8 & 9 & 5 & .037 & 74 & & 28 & 1.03 & 1.02 & 4.0 & 316 \\
\hline & & & & 24 & 88 & & & 1.1 & 0.99 & 23.2 & 128 \\
\hline & & & & 1 & 1 & & & 1.0 & 1.00 & & 593 \\
\hline & & & & & & & & 0.9 & 0.88 & 7.0 & 25 \\
\hline & & & & & & & & 1. & 1.20 & 9.0 & 0 \\
\hline & & & & & & & & 1.11 & 1.00 & 7.0 & 580 \\
\hline $\mathrm{H}$ & & & 0.5 & & & & & 1.21 & 1.07 & 5.0 & 272 \\
\hline $2 / 55$ & 7.2 & 4 & & & & & & 0 & 75 & 26.3 & 427 \\
\hline $\mathrm{H}$ & & & & & & & & 0.99 & 0.94 & 6.6 & 487 \\
\hline HIF & & & & & & & & 1.01 & 01 & .8 & 60 \\
\hline $\mathrm{H}$ & & & & & & & & 3 & 01 & .3 & 0 \\
\hline HIF & & & & & & 4. & & 0.9 & 0.93 & 7.4 & 0 \\
\hline /34 & \pm & \pm & 0.6 & 6 & 78 & $4.30 \pm$ & 11 & 0.97 & 0.9 & 23.5 & 693 \\
\hline 446 & \pm( & 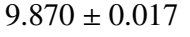 & 0.52 & 97 & 81 & $5=$ & 24 & 1.03 & 0.86 & 7.9 & 498 \\
\hline /02 & 6 & 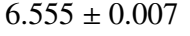 & & 3 & 37 & $4.53=$ & 5 & 1.10 & 1.13 & 11.8 & 287 \\
\hline $\mathrm{HIl}$ & \pm & + & 0 & \pm & 8 & 4.0 & 9 & 1.19 & 0.93 & 6.0 & 227 \\
\hline & $7+1$ & 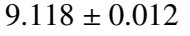 & .48 & $0.5^{5}$ & 1 & $3.13 \pm$ & 1 & 1.06 & 0 & 1.9 & 583 \\
\hline $\mathrm{H}$ & $70+0$ & 6.73 & & & 2 & 3. & 8 & 1.24 & 09 & 4.7 & 147 \\
\hline HIF & +8 & 5.2 & 0.87 & & 07 & 6.1 & 2 & 0.78 & 0.78 & 4.7 & 28 \\
\hline HIP 1 & $9+8$ & 8.47 & & & & 4. & 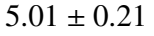 & 0.98 & 0.98 & 4.0 & 199 \\
\hline HIP 1 & + & 00 & & & & & .21 & 1.06 & 0.90 & 4.5 & 235 \\
\hline HIP 1 & & 0 & 0.638 & $0.714 \pm 0.060$ & $20.87 \pm 1.99$ & $4.67 \pm 0$ & $4.71 \pm 0.21$ & 1.06 & 1.05 & 4.0 & 198 \\
\hline HIP 114914 & $8.174 \pm 0.006$ & $8.547 \pm 0.008$ & $0.638 \pm 0.029$ & 0.044 & $18.30 \pm 1.97$ & $4.39 \pm 0.23$ & $4.76 \pm 0.23$ & 1.09 & 1.04 & 5.3 & 301 \\
\hline
\end{tabular}

rotational velocities and of the chromospheric $\mathrm{Ca}$ II H\&K emission is presented.

Pairs for which at least one of the components is a doublelined spectroscopic binary or which show rotational velocity higher than about $10 \mathrm{~km} \mathrm{~s}^{-1}$ were not included in this study because very high-precision abundance analysis is not possible for them. The sample considered in this paper consists of 33 pairs.

\section{Stellar properties}

Table 1 reports visual and absolute magnitudes, $B-V$ colors, parallaxes, masses, and projected separations for the program stars. As in Paper I, Hipparcos and Tycho photometries were averaged and corrected to the standard system using the calibrations by Bessell (2000). The parallaxes are from Hipparcos. When separate parallaxes were available for the two components, a weighted average was performed. The absolute magnitudes were corrected for the Lutz-Kelker effect as in Paper I.

The stellar masses were determined from the isochrones of Girardi et al. (2002) by averaging the masses obtained for $1 \mathrm{Gyr}$ main-sequence stars with absolute magnitudes and temperatures equal to those of the program stars for the appropriate metallicity. To take the dependencies on temperature and metallicity into account, the masses were determined iteratively during the abundance analysis. For the pairs whose primaries show significant evolution out of the main sequence (namely HIP 3290,
HIP 74432, HIP 80399), stellar masses were taken from the suitable isochrone that fits the position of the components on the color-magnitude diagram.

Table 2 summarizes the magnitude differences for program stars, including Hipparcos, Tycho, and further ground-based photometry when available. Only literature observations reported in the standard system are included. Error estimates are assigned as in Paper I. We note that in several cases the available magnitude differences show discrepancies of $0.05-0.10$ mag, and even of $0.34 \mathrm{mag}$ in the worst case (HIP 76446). This has a significant impact on the analysis of some of the pairs with suspected abundance differences discussed in this work. New, high precision differential photometry would be useful in these cases.

\section{Abundance analysis}

\subsection{Equivalent widths}

Equivalent widths (hereafter $E W \mathrm{~s}$ ) were measured on monodimensional spectra using an automatic procedure that estimates a local continuum level and performs a Gaussian fit to the selected lines (see Bragaglia et al. 2001, for details).

The line list is the same one used in Paper I, with a small extension to the blue because of the wider spectral coverage of FEROS spectra. We did not use lines with the wavelength lower 


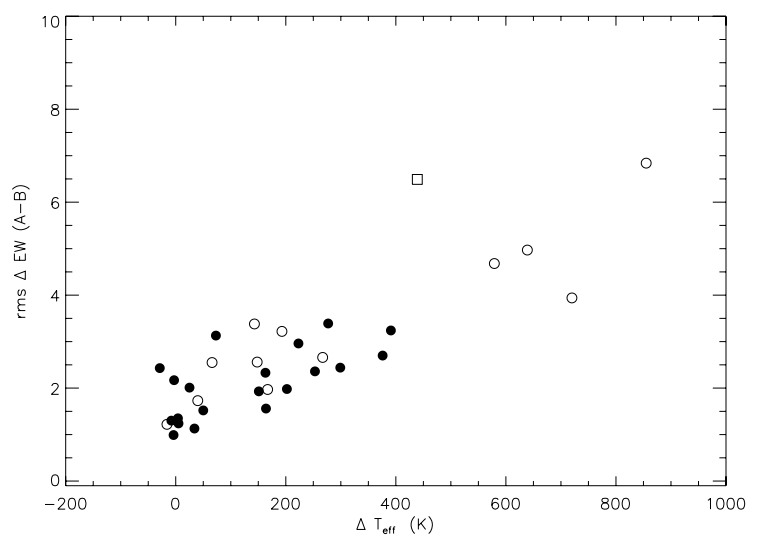

Fig. 1. The rms scatter in equivalent-width differences $\Delta E W$ between the components of the binaries analyzed in this paper as a function of temperature difference $\Delta T_{\text {eff }}$. Open circles represent pairs with a temperature difference larger than $400 \mathrm{~K}$, and the primary clearly evolved off the main sequence and active stars. Filled circles represent the other pairs. The anomalously large scatter in $E W$ difference for HIP 64030 (plotted as an open square) is probably due to the large abundance difference between the components.

than 4500 A because of the severe blending and difficulty in continuum tracing.

Internal errors of $E W s$ can be estimated by the rms in the difference of $E W \mathrm{~s}$ between the components of pairs whose temperatures differ by less than $200 \mathrm{~K}$, about $2 \mathrm{~mA}$ (Fig. 1). The absolute errors of $E W \mathrm{~s}$ are larger. They can be estimated by considering the 6 pairs in common with those studied in Paper I. The $E W$ s show an offset, $\left(E W_{\text {SARG }}=E W_{\text {FEROS }}-(3.537+0.121 \times\right.$ $\left.E W_{\mathrm{FEROS}}\right)$ and $E W_{\text {SARG }}=E W_{\mathrm{FEROS}}-\left(4.167+0.077 \times E W_{\mathrm{FEROS}}\right)$ for the two observing runs), with FEROS $E W s$ being larger (Fig. 2). The dispersion of the $E W \mathrm{~s}$ difference from these relations is about $6.0 \mathrm{~m} \AA$. We were not able to identify the source of this discrepancy, possibly due the different spectral resolution or to the different background subtraction.

For our analysis, we chose to adopt the SARG system and then we applied the measured offset, separately for the two observing runs, to FEROS $E W \mathrm{~s}$, The final equivalent widths are reported in Table 3, available only in electronic form at the CDS.

\subsection{Analysis of primaries}

The analysis technique is the same as used in Paper I and we describe it here in some detail. The primaries were analyzed differentially with respect to the Sun. An automatic procedure was used to iteratively remove outliers that yield abundances differing more than $2.5 \sigma$ from the average of remaining lines.

The atmospheric parameters of the primaries were derived iteratively as follows:

- effective temperature was determined from the ionization equilibrium of iron and the difference VI vs. Fe II. At any iteration, the change in the effective temperature required for Fe I vs. Fe II and VI vs. Fe II is given by

$$
\begin{aligned}
& \Delta T_{\text {eff }}(\mathrm{Fe} \mathrm{I}-\mathrm{Fe} \text { II })=-(A(\mathrm{Fe} \mathrm{I})-A(\mathrm{Fe} I \mathrm{II})-0.050) / S_{\mathrm{Fe}} \\
& \Delta T_{\text {eff }}(\mathrm{VI}-\mathrm{Fe} \mathrm{II})=-(A(\mathrm{VI})-A(\mathrm{Fe} \mathrm{II})+3.52) / S_{V}
\end{aligned}
$$

where $A(\mathrm{Fe} \mathrm{I}), A(\mathrm{Fe} \mathrm{II})$, and $A(\mathrm{VI})$ are the abundance of Fe I, Fe II, and VI respectively; $S_{\mathrm{Fe}}=0.0009 \mathrm{dex} / \mathrm{K}$ and $S_{V}=$ $0.0015 \mathrm{dex} / \mathrm{K}$ are the slopes of the $T_{\text {eff }}$ vs. (Fe I-Fe II) and
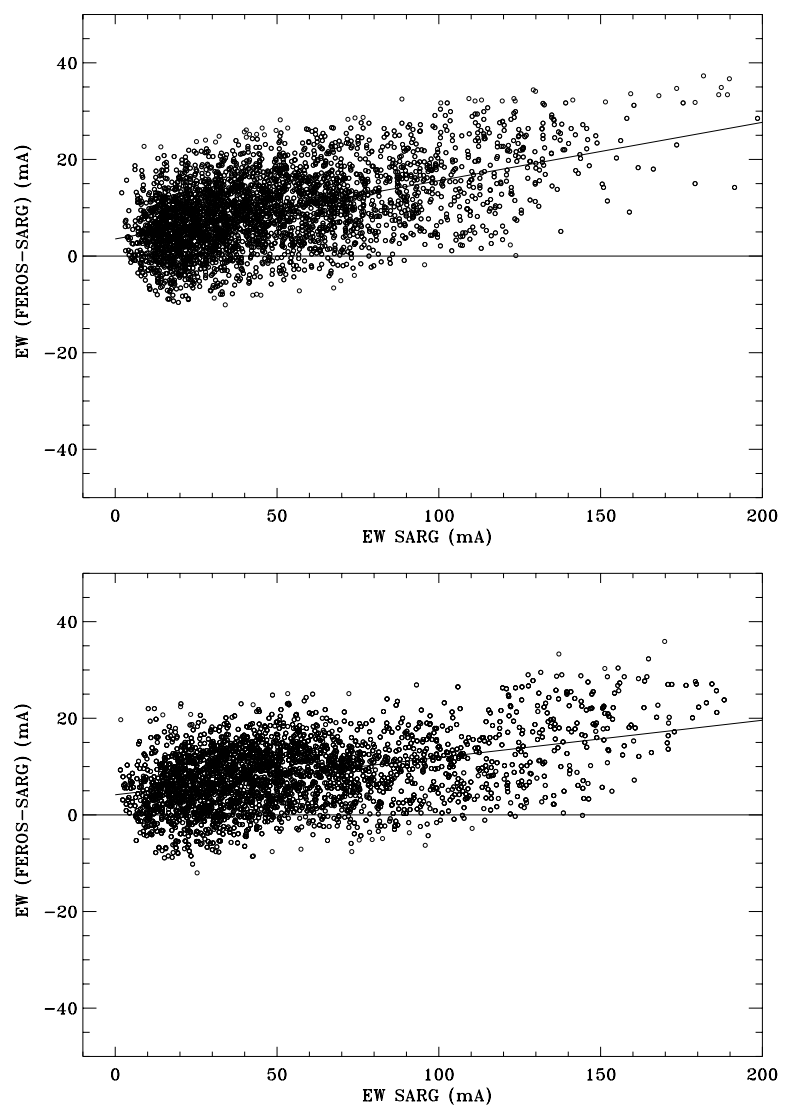

Fig. 2. Comparison of SARG and FEROS EWs. Upper panel: run 1 (August 2001); lower panel: run 2 (March 2002).

$T_{\text {eff }}$ vs. (VI-Fe II) relations for a solar type $\operatorname{star}^{2}$. The two constants 0.050 and 3.52 represent the offset between the mean abundances derived from Fe II and VI lines, with respect to Fe I lines, in the reference solar analysis.

The high temperature sensitivity of $\mathrm{V}$ lines allows us to achieve better precision for determining the temperature difference. Details on the inclusion of the hyperfine splitting of $\mathrm{V}$ lines are given in Paper I and references therein.

The adopted temperature difference at any iteration is given by the weighted average of $\Delta T_{\text {eff }}(\mathrm{Fe} I-\mathrm{Fe}$ II $)$ and $\Delta T_{\text {eff }}(\mathrm{VI}-\mathrm{Fe}$ II);

- stellar gravities were calculated from the relation (see e.g. Nissen et al. 1997)

$\log g=$ const. $+\log M+0.4\left(M_{V}+B C\right)+4 \log T_{\text {eff }}$

where the constant term includes the solar quantities. We used the absolute magnitudes from Hipparcos parallaxes, the stellar masses determined from the isochrones of Girardi et al. (2002), including the dependence of masses on metallicity, and the bolometric corrections by Kurucz (1995). Since the derived masses and gravities depend on temperatures and metallicities, an iterative procedure is required;

- stellar metallicity in the atmospheric model was assumed to be equal to the abundance of Fe I resulting from the analysis; - microturbulent velocity adopted in the final analysis was derived, as in Paper I, from a relation between microturbulent velocity and effective temperature: $\xi=-5.98+0.00122 T_{\text {eff }}$.

2 The slopes actually have a small dependence on effective temperature, but the impact of this on our results is negligible because of the use of an iterative procedure until convergence. 
The microturbulent velocities used to derive this relation were based on a first tentative analysis, where the microturbulent velocities were modified in order to cancel any trend of abundances with expected line strength (see Magain 1984). Excluding a few outliers (evolved stars), the dispersion around the relation is $0.18 \mathrm{~km} \mathrm{~s}^{-1}$. This relation is different with respect to that of Paper I, probably because of the different roles played by the deviations from a Gaussian profile by the actual spectral line-profile determined from spectra taken at different resolutions coupled with the use of a Gaussian fitting routine to extract $E W$ s. For a few pairs that include evolved stars the microturbulence was instead determined individually by eliminating the trend of iron abundance with expected $E W$.

As the atmospheric parameters are not independent of each other, an iterative procedure was required. This was performed in a nearly automatic way by calculating the temperature of the successive iteration from the relations Eqs. (1), (2) and deriving the other atmospheric parameters from the new temperature and the iron abundance of the previous run. Convergence was usually achieved in about ten iterations. We checked that the final result was robust against different starting conditions.

In a few cases (HIP 3290, HIP 58862/4, HIP 76603/2, HIP 79818), for which Hipparcos parallaxes gave absolute magnitudes for the two components inconsistent with any single isochrone, we adopted spectroscopic distances that are derived by using effective temperatures from the excitation equilibrium and gravity from the ionization equilibrium for iron $(d=84 \mathrm{pc}$ for HIP 3290; $d=45$ pc for HIP 55288; $d=103$ pc for HIP $58862 / 4 ; d=36$ pc for HIP $76603 / 2 ; d=82$ pc for HIP 79818). The location of the components of these binaries in the HR diagram and in the $T_{\text {eff }}-\log g$ diagram using these distances matched single isochrones much better than did the location obtained from the Hipparcos parallaxes (see Fig. 3).

Note that this method is optimized for the differential analysis, for which errors in the distance are of no concern (at least for pairs with a small temperature difference, see Sect. 4.4). In the analysis of primaries, this source of errors is instead relevant, especially considering that the binarity of our program stars often induces large parallax errors. This effect is clearly seen in Fig. 3: adopting a fainter absolute magnitude gives a higher gravity (Eq. (3)), and then a warmer temperature derived through ionization equilibrium. For a difference of one magnitude, the effect is about $200 \mathrm{~K}$. The warmer temperature will yield larger microturbulent velocities (by about $0.25 \mathrm{~km} \mathrm{~s}^{-1}$ ) and metallicities (by $0.10-0.15$ dex); i.e. all the atmospheric parameters are significantly affected.

Table 4 summarizes the atmospheric parameters adopted in the abundance analysis. This table also lists the atmospheric parameters of the secondaries, derived as part of the differential analysis described in Sect. 4.3. Table 5 shows the results of the analysis of the primaries.

\subsection{Differential analysis of secondaries}

The secondary of each pair was analyzed differentially with respect to the primary, strictly using the same line set. The initial line list for the differential analysis included the lines not removed as outliers in the analysis of both components. Further iterative clipping was performed to exclude lines that gave abundance differences outside $\pm 2.5 \sigma$ with respect to the average of the other lines. The whole analysis was performed working on line-by-line abundance differences between the primary and the
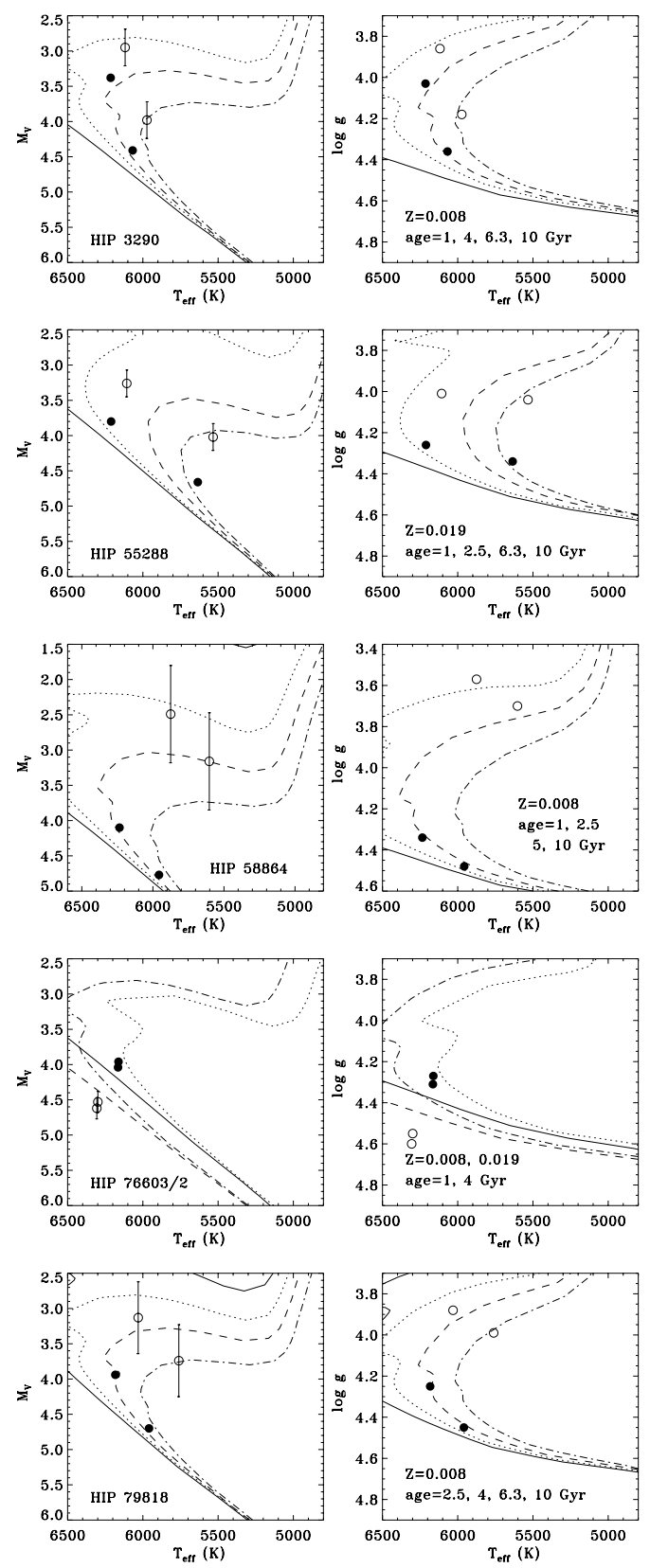

Fig. 3. Position of the components of the pairs for which Hipparcos parallaxes yielded inconsistent position in the HR diagram (left panel) and in the $T_{\text {eff }}-\log g$ diagram (right panel). Open circles: results of abundance analysis performed adopting the Hipparcos distance; filled circles: adopting spectroscopic distances. Isochrones by Girardi et al. (2002) of suitable ages and metallicties are overplotted. From top to bottom: HIP 3290, HIP 55288; HIP 58864/2; HIP 76603/2, HIP 79818.

secondary, therefore removing systematic errors that affect the abundance of a single line in a similar way for both components (e.g. $\log g f$ values, part of the errors on $E W$ measurement, etc.). The atmospheric parameters of the secondaries were derived as differences with respect to those of the primaries (except for the microturbulence, adopted from the relation introduced in Sect. 4.2, as for the primaries):

- the difference of effective temperature with respect to the primary was derived using Eqs. (1), (2) in a differential way

$$
\begin{aligned}
& \Delta T_{\mathrm{eff}}(\mathrm{Fe} \mathrm{I}-\mathrm{Fe} \mathrm{II})=-\left[\left(A_{\mathrm{Fe} I}-A_{\mathrm{Fe} \mathrm{III}}\right)_{\mathrm{A}}-\left(A_{\mathrm{Fe} \mathrm{I}}-A_{\mathrm{Fe} \mathrm{III}}\right)_{\mathrm{B}}\right] / S_{\mathrm{Fe}}(4) \\
& \Delta T_{\mathrm{eff}}(\mathrm{VI}-\mathrm{Fe} \mathrm{II})=-\left[\left(A_{\mathrm{VI}}-A_{\mathrm{Fe} \mathrm{II}}\right)_{\mathrm{A}}-\left(A_{\mathrm{VI}}-A_{\mathrm{Fe} I \mathrm{II}}\right)_{\mathrm{B}}\right] / S_{V}(5)
\end{aligned}
$$


Table 4. Adopted atmospheric parameters.

\begin{tabular}{|c|c|c|c|c|c|c|c|c|}
\hline Object & $T_{\mathrm{eff}(\mathrm{A})}$ & $T_{\text {eff(B) }}$ & $\log g_{\mathrm{A}}$ & $\log g_{\mathrm{B}}$ & {$[\mathrm{A} / \mathrm{H}]_{\mathrm{A}}$} & {$[\mathrm{A} / \mathrm{H}]_{\mathrm{B}}$} & $\xi_{\mathrm{A}}$ & $\xi_{\mathrm{B}}$ \\
\hline HIP 3290 & 6193 & 6049 & 4.03 & 4.35 & -0.42 & -0.36 & 1.60 & 1.43 \\
\hline HIP 10175 & 5638 & 5338 & 4.33 & 4.36 & -0.08 & -0.08 & 0.92 & 0.56 \\
\hline HIP 20552 & 5880 & 5626 & 4.45 & 4.49 & 0.02 & 0.05 & 1.22 & 0.91 \\
\hline HIP 37923 & 5404 & 5337 & 4.54 & 4.54 & 0.18 & 0.12 & 0.64 & 0.56 \\
\hline HIP 39409 & 5527 & 5499 & 4.35 & 4.36 & 0.02 & 0.01 & 0.79 & 0.75 \\
\hline HIP 44817 & 5384 & 5121 & 4.43 & 4.41 & 0.23 & 0.20 & 0.61 & 0.29 \\
\hline HIP 49520 & 5890 & 5839 & 4.58 & 4.62 & -0.23 & -0.22 & 1.23 & 1.17 \\
\hline HIP 51266 & 5356 & 5207 & 4.60 & 4.61 & 0.01 & 0.01 & 0.58 & 0.40 \\
\hline HIP 55288 & 6211 & 5635 & 4.26 & 4.34 & 0.06 & 0.08 & 1.63 & 0.92 \\
\hline HIP 56280 & 6280 & 6206 & 4.17 & 4.17 & 0.01 & 0.02 & 1.71 & 1.62 \\
\hline HIP 58298 & 6015 & 5980 & 4.09 & 4.11 & -0.56 & -0.56 & 1.39 & 1.34 \\
\hline HIP 58813 & 5900 & 5523 & 4.32 & 4.41 & 0.07 & 0.06 & 1.24 & 0.78 \\
\hline HIP 58864 & 6235 & 5957 & 4.34 & 4.48 & -0.36 & -0.35 & 1.65 & 1.31 \\
\hline HIP 59743 & 5232 & 5073 & 4.31 & 4.32 & 0.01 & 0.00 & 0.43 & 0.23 \\
\hline HIP 62596 & 6134 & 6163 & 4.33 & 4.33 & 0.05 & 0.09 & 1.53 & 1.57 \\
\hline HIP 64030 & 6197 & 5757 & 3.88 & 3.91 & -0.62 & -0.35 & 1.61 & 1.07 \\
\hline HIP 65176 & 6201 & 5804 & 4.38 & 4.51 & 0.10 & 0.07 & 1.61 & 1.13 \\
\hline HIP 65352 & 5141 & 4936 & 4.61 & 4.60 & -0.43 & -0.45 & 0.32 & 0.06 \\
\hline HIP 69328 & 5753 & 5589 & 4.12 & 4.20 & -0.30 & -0.29 & 1.06 & 0.86 \\
\hline HIP 70269 & 5909 & 5904 & 4.32 & 4.33 & -0.29 & -0.30 & 1.26 & 1.25 \\
\hline HIP 70386 & 6112 & 5918 & 4.52 & 4.51 & -0.03 & -0.06 & 1.50 & 1.27 \\
\hline HIP 73674 & 5745 & 5739 & 4.46 & 4.46 & -0.33 & -0.36 & 1.05 & 1.05 \\
\hline HIP 74432 & 5612 & 5444 & 4.16 & 4.40 & 0.03 & 0.03 & 0.92 & 0.51 \\
\hline HIP 76446 & 5810 & 5090 & 4.40 & 4.50 & -0.10 & -0.05 & 1.13 & 0.25 \\
\hline HIP 76602 & 6162 & 6165 & 4.27 & 4.31 & -0.25 & -0.16 & 1.57 & 1.57 \\
\hline HIP 78024 & 6154 & 5298 & 4.35 & 4.58 & 0.06 & 0.10 & 1.56 & 0.51 \\
\hline HIP 79818 & 6183 & 5959 & 4.25 & 4.45 & -0.43 & -0.34 & 1.59 & 1.32 \\
\hline HIP 80399 & 6016 & 6032 & 4.08 & 4.33 & 0.08 & 0.10 & 1.75 & 1.41 \\
\hline HIP 84405 & 4975 & 4979 & 4.52 & 4.53 & -0.32 & -0.32 & 0.11 & 0.12 \\
\hline HIP 101292 & 5488 & 5496 & 4.39 & 4.40 & 0.04 & 0.03 & 0.74 & 0.75 \\
\hline HIP 103438 & 5840 & 5201 & 4.50 & 4.40 & 0.00 & 0.02 & 1.55 & 0.39 \\
\hline HIP 104687 & 5781 & 5740 & 4.41 & 4.41 & 0.05 & 0.06 & 1.10 & 1.05 \\
\hline HIP 114914 & 5826 & 5674 & 4.32 & 4.40 & 0.06 & 0.07 & 1.15 & 0.97 \\
\hline
\end{tabular}

and then removing the possible source of errors caused by the constant terms in Eqs. (1), (2) (that might be due to e.g. a non solar $[\mathrm{V} / \mathrm{Fe}])$.

As for the primaries, the adopted temperature difference is given by the weighted average of $\Delta T_{\mathrm{eff}}(\mathrm{Fe} \mathrm{I}-\mathrm{Fe}$ II) and $\Delta T_{\text {eff }}(\mathrm{VI}-\mathrm{Fe}$ II) (hereafter in short iron and vanadium temperatures).

Internal errors for temperature difference (shown in Table 6) are derived by propagating the uncertainty in the abundances of Fe I, Fe II, and VI due to the line-by-line scatter;

- the difference of stellar gravities was calculated using Eq. (3) in a differential way:

$\left.\Delta \log g(A-B)=\Delta \log M+0.4(\Delta V+\Delta B C)+4 \Delta \log T_{\text {eff }}\right)(6)$

where the terms in Eq. (3) that are equal form the components, including distance and photometric zero point, cancel out when calculating the difference. Therefore, only the error in apparent magnitude difference (Table 2) has to be considered and not the much larger one on the absolute magnitude (Table 1);

- the stellar metallicity in the atmospheric model was assumed as that of the primary plus the Fe I abundance difference between the components;

- microturbulent velocity was taken from the relation introduced in Sect. 4.2, as for the primaries.

As in the case of the primaries, the final parameters were derived by means of an iterative procedure. The internal scatter of

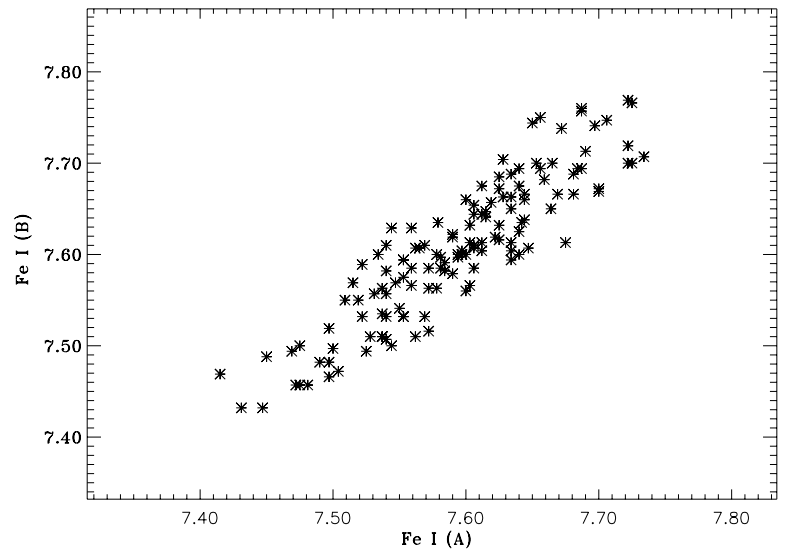

Fig. 4. Iron abundance derived for each line of the components of HIP 114914 A and B. A clear correlation is present, indicating that the use of a line-by-line differential analysis significantly reduces the errors on abundance difference between the components.

abundance differences is much lower than expected, if the errors of the two components were fully independent, indicating that the differential analysis allows removal of a number of possible systematic errors (Fig. 4).

Table 6 lists the temperature differences given by different methods: equilibrium of ionization for $\mathrm{Fe}$, the same replacing VI to $\mathrm{Fe}$ I, the average of the two (the value adopted in our analysis); excitation equilibrium for Fe I lines; magnitude difference 


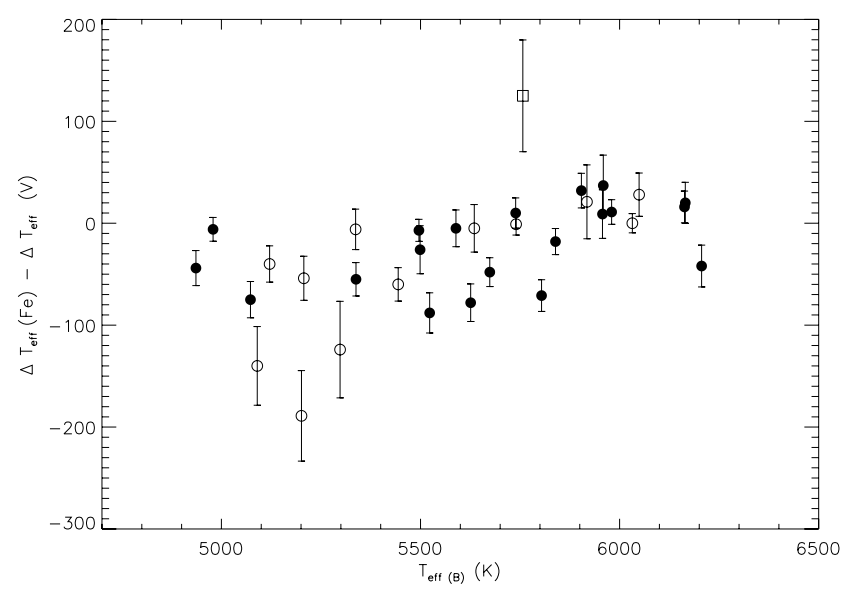

Fig. 5. Difference between the temperature differences based on $\mathrm{Fe}$ and $\mathrm{V}$ lines as a function of the temperature of the secondary. Symbols as in Fig. 1.

(assuming that both components are on main sequence), color temperatures calibrations from $B-V, V-I, V-K, J-K$, and $J-H$ (from Alonso et al. 1996) and $b-y$ (from Nordstrom et al. 2004). When required, transformations between photometric systems were performed as in Paper II. The colors were taken from the following sources: $B-V$ : from Table $1 ; V-I$ : HIP 3290, 44817, 49520, 62596, 65176, 69328, 114914: from Cuypers \& Seggewiss (1999); HIP 37923, 58815, 58864, 65352, 74432, 76603: from Hipparcos (ESA 1997); HIP 103438: from Zuckerman et al. (2001); $b-y$ : from Nordstrom et al. (2004); $J, H, K: 2 \mathrm{MASS}$ all sky release (Cutri et al. 2003). The temperature difference from the magnitude difference $\Delta V$ (taken from Table 2) is estimated by considering the slope of the main sequence on the $1 \mathrm{Gyr}$ solar metallicity isochrone. It does not include evolutionary effects and therefore represents an upper limit to the actual temperature difference.

Figure 5 shows the comparison between the temperature differences based on Fe and V lines as a function of the temperature of the secondary. A similar trend was obtained in Paper I from SARG data. Therefore, it seems that some systematic trend in the two-temperature scales appears below $5500 \mathrm{~K}$.

This might be explained by considering that $\mathrm{V}$ lines are formed mostly in atmospheric regions outside those where Fe I (and Fe II) lines form. Therefore the derived V abundance is very sensitive to the temperature gradient in the external parts of the stellar atmosphere. If the temperature gradient in the adopted 1-D model is too shallow, as suggested by comparison with 3D models (Asplund 2005), then a lower effective temperature is derived. An alternative possibility is represented by deviations from local thermodynamical equilibrium (Bodaghee et al. 2003).

For a few pairs, characterized by large temperature differences, or involving active stars or with evolved primaries, the analysis depended on the adopted microturbulent velocity (mean relation vs optimization) and on the maximum $E W$ of lines included in it. In a few cases, leaving microturbulence as a free parameter eliminates any trend of abundance difference with $E W$, but also introduces a significant trend of abundance difference with excitation potential. To reduce these uncertainties in all these problematic cases (except for HIP 80399A and HIP 74432A, clearly evolved off of the main sequence), we adopted the microturbulent velocity from the standard relation but we included in the analysis only those lines with an expected equivalent width smaller than about $60 \mathrm{~m} \AA$.

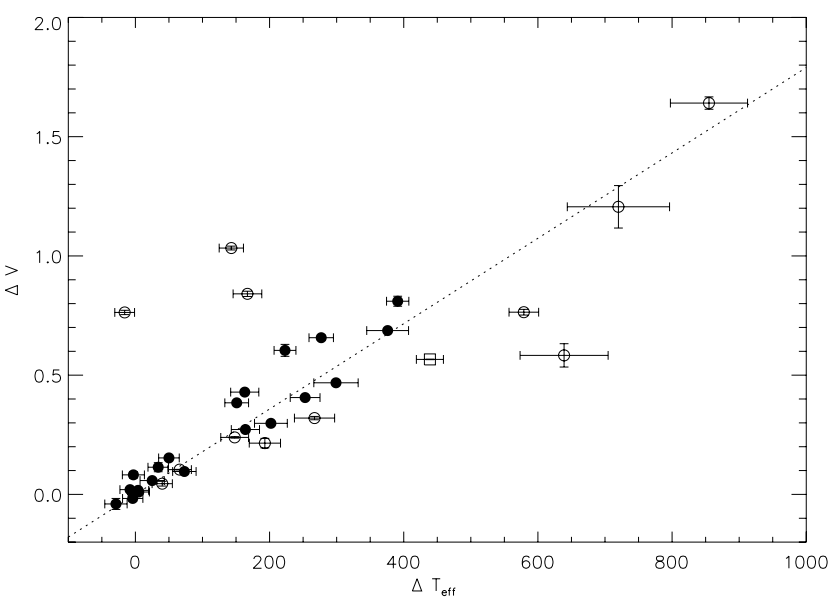

Fig. 6. Magnitude vs. temperature differences. The dotted line represents the typical slope along the main sequence of a 1 Gyr solarmetallicity isochrone. Symbols as in Fig. 1. The three stars clearly above the mean relation are HIP 3290, HIP 74332, and HIP 80399, which all have primaries evolved off of the main sequence. The pair with a magnitude difference that is too small for the measured temperature difference is HIP 103438.

The atmospheric parameters of the secondaries are listed in Table 4. Table 7 reports the results of the differential analysis. Plots of abundance difference for Fe I as a function of temperature difference and effective temperature of the primary and of the secondary, as well as of the metallicity are shown in Figs. 7 and 9. The derivation of full error bars as shown in these figures is presented in Sect. 4.4. Final abundance and temperature differences (including the full error bars) are listed in Table 11.

\subsection{Errors analysis}

A careful error analysis is mandatory for evaluating the significance of several small abundance differences found in our analysis. We first consider the errors that directly affect the differential analysis, and then we discuss the indirect impact of absolute errors.

The errors due to line-by-line scatter (listed in Table 7) are always below 0.01 dex. The uncertainties in the determinations of the atmospheric parameters were also estimated. To propagate these errors into errors on abundance and temperature difference, we derived the sensitivity to abundance and temperature difference into changes of atmospheric parameters for a few selected pairs with effective temperatures spanning the range of the program stars (Table 8). Table 9 shows the adopted relations.

Errors on $\Delta T_{\text {eff }}$ include the errors due to line-by-line scatter of Fe I, Fe II, and VI (Table 6) and the errors in the other atmospheric parameters. Errors on $\Delta \log g$ include the errors in the magnitude difference between the components (Table 2), the errors on $\Delta T_{\text {eff }}$, and the errors on $\triangle \log M$ and $\triangle B C$. The error on $\Delta \log M$ from assuming the adopted main sequence references caused by the errors on $\Delta T_{\text {eff }}, \Delta[\mathrm{Fe} / \mathrm{H}]$ and $\Delta V$ is below 0.01 dex. The same also holds for error on bolometric correction. To take into account the additional uncertainty due to e.g. evolutionary effects a further 0.02 dex error on $\Delta \log g$ was conservatively included. Errors on $\Delta[\mathrm{A} / \mathrm{H}]$ were assumed to be the same as the full errorbar on $\Delta[\mathrm{Fe} / \mathrm{H}]$.

The uncertainty in the microturbulent velocity was estimated by considering the rms of the difference (between the components) of the residuals from the $\xi$ vs. $T_{\text {eff }}$ relation. It results $0.15 \mathrm{~km} \mathrm{~s}^{-1}$, smaller than the expectation from fully 
Table 7. Results of differential analysis.

\begin{tabular}{|c|c|c|c|c|c|c|c|c|c|}
\hline$\overline{\text { Object }}$ & 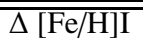 & $\overline{\mathrm{rms}}$ & 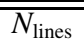 & 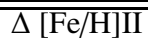 & $\overline{\mathrm{rms}}$ & 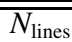 & 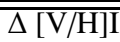 & $\overline{\mathrm{rms}}$ & 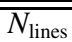 \\
\hline HIP 3290 & -0.056 & 0.085 & 103 & -0.046 & 0.057 & 29 & -0.021 & 0.072 & 10 \\
\hline HIP 10175 & -0.002 & 0.043 & 123 & -0.034 & 0.059 & 27 & -0.063 & 0.046 & 21 \\
\hline HIP 20552 & -0.032 & 0.036 & 123 & -0.071 & 0.068 & 31 & -0.122 & 0.065 & 20 \\
\hline HIP 37923 & 0.059 & 0.053 & 61 & 0.056 & 0.058 & 23 & 0.052 & 0.091 & 33 \\
\hline HIP 39409 & 0.000 & 0.040 & 130 & -0.016 & 0.102 & 34 & -0.028 & 0.058 & 25 \\
\hline HIP 44817 & 0.032 & 0.029 & 34 & 0.014 & 0.056 & 23 & -0.017 & 0.067 & 26 \\
\hline HIP 49520 & -0.012 & 0.035 & 134 & -0.018 & 0.041 & 33 & -0.033 & 0.047 & 12 \\
\hline HIP 51266 & 0.003 & 0.048 & 107 & -0.030 & 0.079 & 25 & -0.055 & 0.054 & 26 \\
\hline HIP 55288 & -0.018 & 0.082 & 86 & -0.016 & 0.029 & 18 & -0.027 & 0.126 & 18 \\
\hline HIP 56280 & -0.006 & 0.071 & 129 & -0.025 & 0.058 & 27 & -0.058 & 0.055 & 8 \\
\hline HIP 58298 & 0.003 & 0.033 & 113 & 0.002 & 0.023 & 29 & 0.016 & 0.055 & 11 \\
\hline HIP 58813 & 0.013 & 0.045 & 119 & -0.046 & 0.091 & 35 & -0.081 & 0.017 & 11 \\
\hline HIP 58864 & -0.013 & 0.058 & 117 & -0.011 & 0.066 & 34 & -0.002 & 0.100 & 12 \\
\hline HIP 59743 & 0.009 & 0.032 & 125 & -0.026 & 0.069 & 31 & -0.081 & 0.068 & 30 \\
\hline HIP 62596 & -0.041 & 0.049 & 121 & -0.034 & 0.049 & 28 & -0.022 & 0.057 & 16 \\
\hline HIP 64030 & -0.268 & 0.067 & 96 & -0.263 & 0.070 & 36 & -0.084 & 0.225 & 8 \\
\hline HIP 65176 & 0.028 & 0.057 & 131 & -0.009 & 0.052 & 30 & -0.054 & 0.047 & 10 \\
\hline HIP 65352 & 0.019 & 0.029 & 109 & -0.010 & 0.052 & 17 & -0.028 & 0.042 & 27 \\
\hline HIP 69328 & -0.007 & 0.054 & 142 & -0.009 & 0.049 & 32 & -0.013 & 0.093 & 19 \\
\hline HIP 70269 & 0.009 & 0.032 & 117 & 0.016 & 0.041 & 32 & 0.055 & 0.084 & 15 \\
\hline HIP 70386 & 0.032 & 0.075 & 125 & 0.040 & 0.103 & 26 & 0.058 & 0.148 & 15 \\
\hline HIP 73674 & 0.032 & 0.031 & 133 & 0.037 & 0.058 & 33 & 0.045 & 0.048 & 15 \\
\hline HIP 74432 & 0.004 & 0.049 & 122 & -0.030 & 0.065 & 34 & -0.064 & 0.060 & 28 \\
\hline HIP 76446 & -0.051 & 0.064 & 74 & -0.131 & 0.136 & 25 & -0.208 & 0.108 & 22 \\
\hline HIP 76602 & -0.087 & 0.041 & 120 & -0.083 & 0.044 & 30 & -0.058 & 0.089 & 12 \\
\hline HIP 78024 & -0.039 & 0.094 & 115 & -0.065 & 0.199 & 27 & -0.181 & 0.144 & 12 \\
\hline HIP 79818 & -0.087 & 0.048 & 93 & -0.085 & 0.024 & 25 & -0.033 & 0.131 & 9 \\
\hline HIP 80399 & -0.022 & 0.025 & 92 & -0.022 & 0.038 & 30 & -0.021 & 0.019 & 16 \\
\hline HIP 84405 & 0.001 & 0.021 & 123 & -0.004 & 0.043 & 20 & -0.005 & 0.014 & 25 \\
\hline HIP 101292 & 0.010 & 0.028 & 125 & 0.004 & 0.047 & 32 & 0.001 & 0.035 & 31 \\
\hline HIP 103438 & -0.019 & 0.075 & 74 & -0.130 & 0.153 & 24 & -0.228 & 0.096 & 16 \\
\hline HIP 104687 & -0.007 & 0.036 & 119 & -0.008 & 0.034 & 34 & -0.008 & 0.055 & 23 \\
\hline HIP 114914 & -0.013 & 0.034 & 134 & -0.033 & 0.051 & 35 & -0.068 & 0.055 & 20 \\
\hline
\end{tabular}

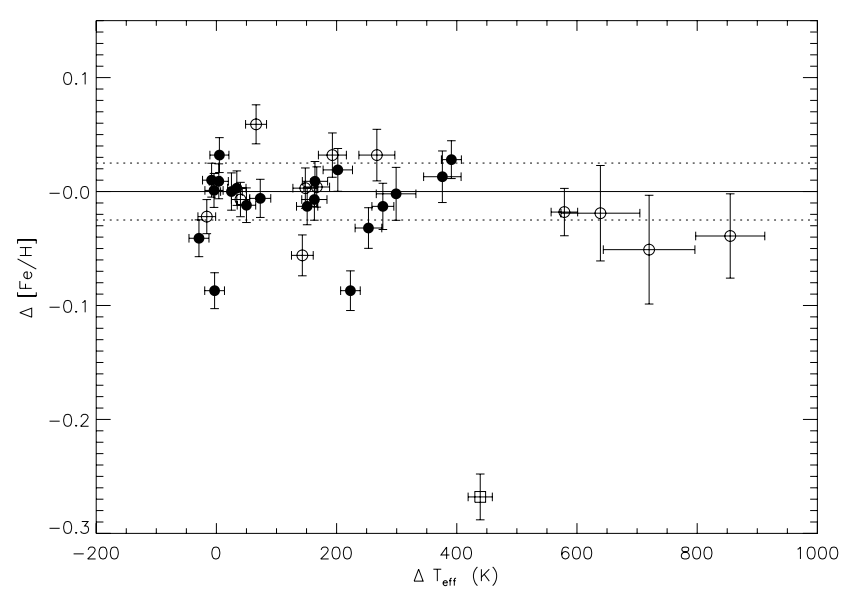

Fig. 7. Iron abundance difference between the components of pairs as a function of the temperature difference. Symbols as in Fig. 1.

independent cases. This indicates that the two components of a pair systematically deviate in the same way from the adopted relation. The typical error on $\Delta \xi$ is then $0.11 \mathrm{~km} \mathrm{~s}^{-1}$. The various error contributions were summed in quadrature, iterating so as to take the dependence of one source from the others into account.

We do not discuss here the absolute errors of the analysis of primaries in detail. Some of them are briefly introduced in Sect. 4.2. Such absolute errors should not be relevant for the differential analysis, as both components are affected in a similar way. However, some indirect effects are still present.
As an example, a wrong parallax would imply a systematically wrong gravity and then a wrong effective temperature derived from ionization equilibrium. We tested these effects by performing the analysis for a few pairs assuming different distances. The analysis was performed in the same way as described in Sects. 4.2 and 4.3, i.e. self-consistently deriving the atmospheric parameters and stellar masses during the analysis. For pairs with similar components $\left(\Delta T_{\text {eff }}<200 \mathrm{~K}\right)$ the effect of a $0.25 \mathrm{mag}$ error on the distance modulus (typical for the program stars) on the abundance difference is less than 0.005 dex. However, for larger temperature differences or larger distance errors they become increasingly important. We derived an approximate relation $\left(\operatorname{err}_{\Delta[\mathrm{Fe} / \mathrm{H}]}=6.0 \times 10^{-5} \Delta T_{\text {eff }} \sigma_{M_{V}}\right)$ to include this source of error in the final abundance difference (Fig. 10).

Other systematic contributions should behave similarly. Errors introduced by the inadequacies of atmospheric models are difficult to estimate. However, as an example of the effectiveness of their minor role for pairs with similar components, we mention the case of HIP 84405, whose components are among the coolest stars in our sample $\left(T_{\text {eff }}<5000 \mathrm{~K}\right.$ for both components). In spite of the probable inadequacies of atmospheric models at low temperatures (see Sect. 4.3), the differential analysis is not affected, because of the similar characteristics of the components.

To include the possible residual effects due to uncertainties in the atmosphere models for pairs with relatively large temperature difference, we used the systematic difference between iron and vanadium temperatures (Fig. 5). For stars with secondaries cooler than $5800 \mathrm{~K}$, the typical slope of the difference 

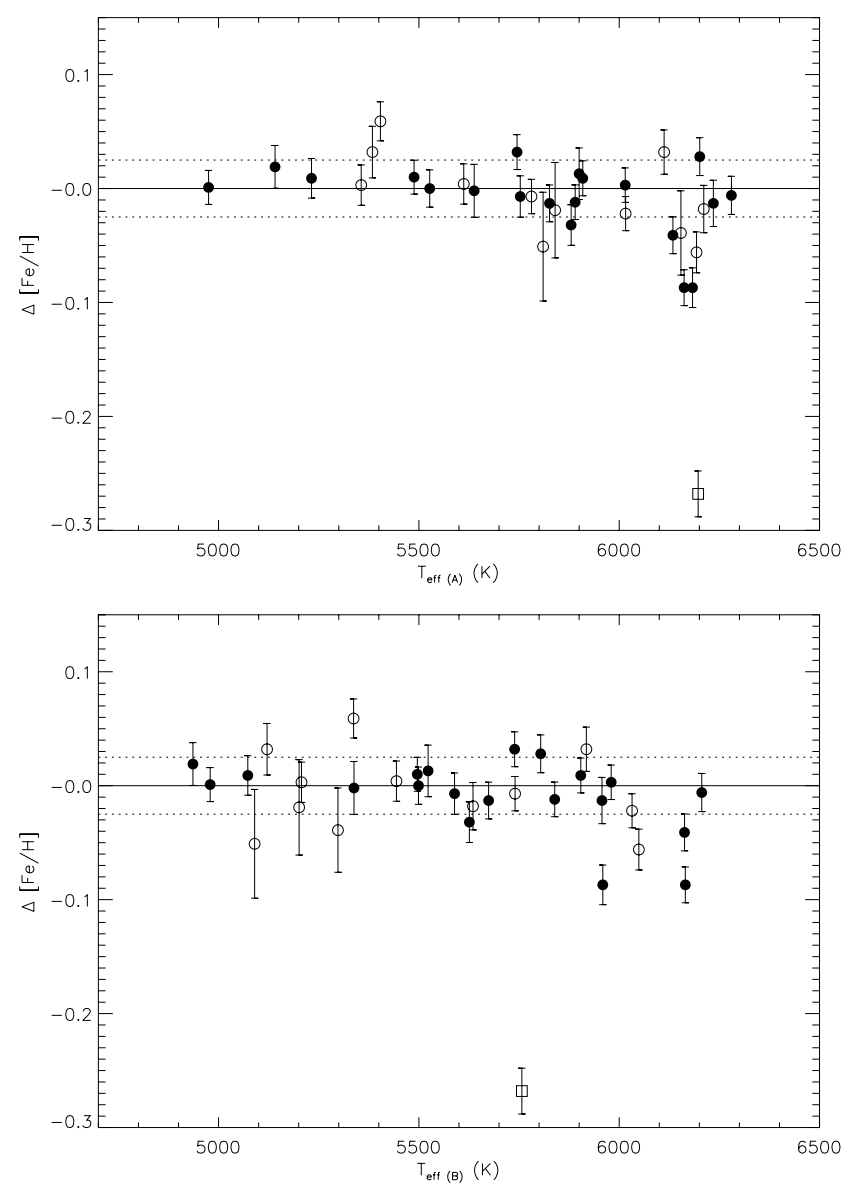

Fig. 8. Iron abundance difference between the components of pairs as a function of the effective temperature of the primary (upper panel) and the secondary (lower panel). Symbols as in Fig. 1.

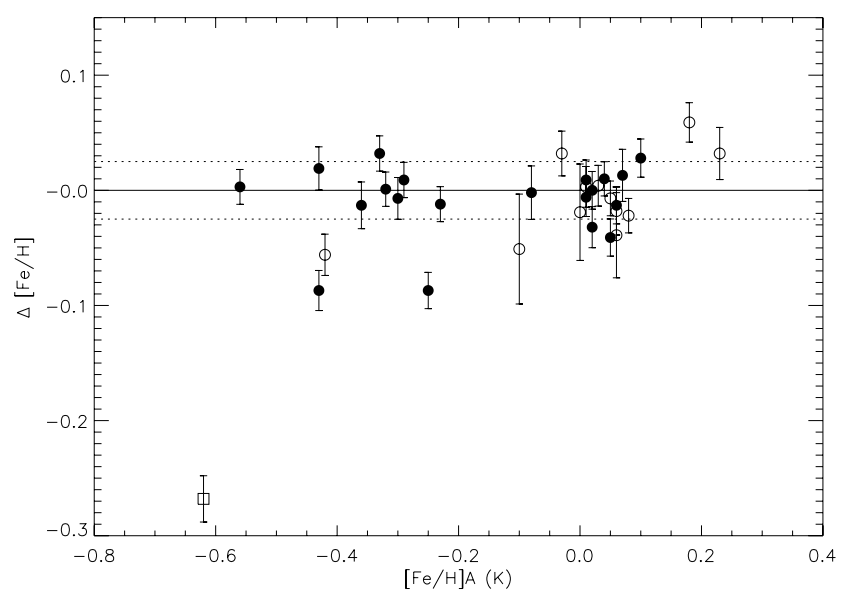

Fig. 9. Iron abundance of the primary between the components of pairs as a function of the metallicity of the primary. Symbols as in Fig. 1.

$\Delta T_{\text {eff }}(\mathrm{Fe}-\mathrm{V})$ vs. the adopted mean $\Delta T_{\text {eff }}$ is about 0.20 . Considering that we averaged the iron and vanadium temperatures, we added a term $\sigma_{T_{\text {eff }}}$ (sys) $=0.10\left(5800-T_{\text {eff }}\right)$ for stars colder than $5800 \mathrm{~K}$. For the warmer stars we considered $\sigma_{T_{\text {eff }}}($ sys $)=10 \mathrm{~K}$. This additional source of error is small for pairs with very similar components but it becomes the dominant source of errors for pairs with $\Delta T_{\text {eff }}>500 \mathrm{~K}$. The observed abundance difference observed for these pairs $(\Delta[\mathrm{Fe} / \mathrm{H}] \sim$ $0.02-0.05 \mathrm{dex})$ is compatible with this error estimate.

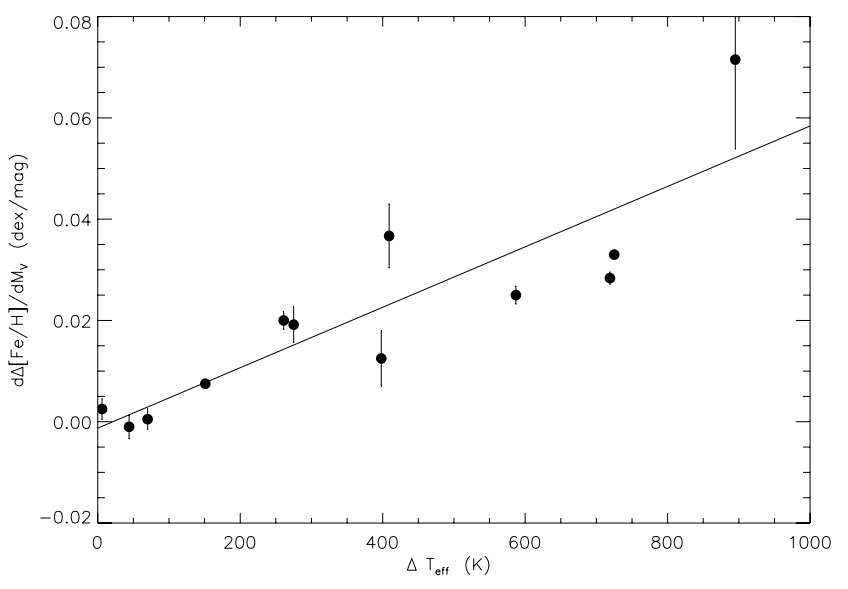

Fig. 10. Estimate of the effect of errors on the absolute magnitude on the differential analysis for pairs as a function of the temperature difference between the components. The abscissa shows the slope of the variation in abundance difference between the components of the pairs as a function of the absolute distance modulus (dex/mag).

The full errorbars of stellar and atmospheric models are larger than the rough estimate given here. However, they are relevant for the differential analysis only if the components of a pair behave in different way with respect to the quantities or physical processes that are the cause of the systematic uncertainties (e.g. helium content, mixing length, etc.), which do not seem plausible when considering similar stars.

It should be noticed that in case of binaries with a real metallicity difference between the components, stellar masses were derived by using stellar models that consider the star chemically uniform, while in case of pollution the stellar interior is more metal poor. From available stellar models with polluted convective zones, we estimated in Paper I that the metal overabundance resulting from assuming unpolluted stellar models is overestimated by less than $10 \%$.

\subsection{Comparison with Paper I}

Comparison of the results of this study and those of Paper I for the six pairs in both samples shows a good agreement. The FEROS-SARG difference of $\Delta T_{\text {eff }}$ between the components is $5 \pm 10 \mathrm{~K}(\mathrm{rms} 23 \mathrm{~K})$ and the FEROS-SARG difference of $\Delta[\mathrm{Fe} / \mathrm{H}]$ between the components is $-0.001 \pm 0.005 \mathrm{dex}$ ( $\mathrm{rms}$ 0.011 dex). While some of the sources of error cancel out in the comparison with Paper I (e.g. model atmosphere, magnitude difference), the small dispersion we found in the difference is an indication of the reliability of the analysis.

\subsection{Individual objects}

We discuss here the objects that show an abundance difference larger than 0.03 dex, which is roughly twice the typical $1 \sigma$ error bar on abundance differences, see Table 11, along with some other peculiar objects. In some cases (such as the pairs with a large temperature difference HIP 78024 and HIP 76446) the abundance difference is actually compatible with our error estimate. Furthermore, when assuming Gaussian distribution of errors, some cases of an abundance differences of 0.03-0.04 dex can be reasonably expected. In specific cases, additional sources of errors might be present (e.g. large activity level, contamination from an unseen companion). While the abundance 
Table 11. Final abundance difference (Fe I from Table 7) and estimates of the iron possibly accreted.

\begin{tabular}{lrrrrrr}
\hline Object & $\Delta T_{\text {eff(A) }}$ & {$[\mathrm{Fe} / \mathrm{H}](\mathrm{A})$} & $\Delta[\mathrm{Fe} / \mathrm{H}]$ & int. Err. & Error & $\Delta M_{\mathrm{Fe}}$ \\
& $\mathrm{K}$ & & & & & $M_{\oplus}$ \\
\hline HIP 3290 & $143 \pm 18$ & -0.42 & -0.056 & 0.008 & 0.018 & $0.33 \pm 0.10$ \\
HIP 10175 & $299 \pm 33$ & -0.08 & -0.002 & 0.004 & 0.023 & $0.08 \pm 0.92$ \\
HIP 20552 & $253 \pm 22$ & 0.02 & -0.032 & 0.003 & 0.018 & $1.01 \pm 0.55$ \\
HIP 37923 & $66 \pm 17$ & 0.18 & 0.059 & 0.007 & 0.017 & $3.66 \pm 1.01$ \\
HIP 39409 & $25 \pm 18$ & 0.02 & 0.000 & 0.004 & 0.016 & $0.00 \pm 0.58$ \\
HIP 44817 & $267 \pm 29$ & 0.23 & 0.032 & 0.005 & 0.023 & $2.82 \pm 1.98$ \\
HIP 49520 & $50 \pm 15$ & -0.23 & -0.012 & 0.003 & 0.015 & $0.13 \pm 0.17$ \\
HIP 51266 & $148 \pm 20$ & 0.01 & 0.003 & 0.005 & 0.018 & $0.15 \pm 0.93$ \\
HIP 55288 & $579 \pm 22$ & 0.06 & -0.018 & 0.009 & 0.021 & $0.60 \pm 0.69$ \\
HIP 56280 & $73 \pm 17$ & 0.01 & -0.006 & 0.006 & 0.017 & $0.09 \pm 0.27$ \\
HIP 58298 & $34 \pm 15$ & -0.56 & 0.003 & 0.003 & 0.015 & $0.01 \pm 0.06$ \\
HIP 58813 & $376 \pm 31$ & 0.07 & 0.013 & 0.004 & 0.023 & $0.30 \pm 0.52$ \\
HIP 58864 & $277 \pm 18$ & -0.36 & -0.013 & 0.005 & 0.020 & $0.11 \pm 0.18$ \\
HIP 59743 & $164 \pm 21$ & 0.01 & 0.009 & 0.003 & 0.017 & $0.49 \pm 0.95$ \\
HIP 62596 & $-29 \pm 16$ & 0.05 & -0.041 & 0.005 & 0.016 & $0.75 \pm 0.29$ \\
HIP 64030 & $439 \pm 20$ & -0.62 & -0.268 & 0.007 & 0.020 & $1.35 \pm 0.07$ \\
HIP 65176 & $391 \pm 16$ & 0.10 & 0.028 & 0.005 & 0.017 & $0.53 \pm 0.31$ \\
HIP 65352 & $202 \pm 24$ & -0.43 & 0.019 & 0.003 & 0.019 & $0.43 \pm 0.42$ \\
HIP 69328 & $163 \pm 20$ & -0.30 & -0.007 & 0.005 & 0.018 & $0.09 \pm 0.23$ \\
HIP 70269 & $4 \pm 15$ & -0.29 & 0.009 & 0.003 & 0.015 & $0.09 \pm 0.16$ \\
HIP 70386 & $193 \pm 23$ & -0.03 & 0.032 & 0.007 & 0.019 & $0.48 \pm 0.29$ \\
HIP 73674 & $5 \pm 15$ & -0.33 & 0.032 & 0.003 & 0.015 & $0.27 \pm 0.13$ \\
HIP 74432 & $167 \pm 21$ & 0.03 & 0.004 & 0.004 & 0.018 & $0.17 \pm 0.75$ \\
HIP 76446 & $720 \pm 76$ & -0.10 & -0.051 & 0.007 & 0.048 & $2.89 \pm 2.69$ \\
HIP 76602 & $-3 \pm 16$ & -0.25 & -0.087 & 0.004 & 0.016 & $0.81 \pm 0.14$ \\
HIP 78024 & $855 \pm 57$ & 0.06 & -0.039 & 0.009 & 0.037 & $2.61 \pm 2.47$ \\
HIP 79818 & $223 \pm 16$ & -0.43 & -0.087 & 0.005 & 0.017 & $0.65 \pm 0.12$ \\
HIP 80399 & $-16 \pm 14$ & 0.08 & -0.022 & 0.003 & 0.015 & $0.59 \pm 0.40$ \\
HIP 84405 & $-4 \pm 15$ & -0.32 & 0.001 & 0.002 & 0.015 & $0.04 \pm 0.56$ \\
HIP 101292 & $-8 \pm 15$ & 0.04 & 0.010 & 0.002 & 0.015 & $0.40 \pm 0.60$ \\
HIP 103438 & $639 \pm 65$ & 0.00 & -0.019 & 0.009 & 0.042 & $1.19 \pm 2.70$ \\
HIP 104687 & $40 \pm 15$ & 0.05 & -0.007 & 0.003 & 0.015 & $0.20 \pm 0.43$ \\
HIP 114914 & $151 \pm 17$ & 0.06 & -0.013 & 0.003 & 0.016 & $0.41 \pm 0.51$ \\
\hline & & & & & &
\end{tabular}

difference derived for these objects might be spurious, a dedicated discussion is useful here.

- HIP 64030. HIP $64030=$ HD 113984 shows a large abundance difference, with the secondary more metal rich by about $0.27 \mathrm{dex}$, that is, about 15 times its error bar. Performing the analysis with different assumptions (different absolute magnitude, optimization of microturbulent velocity instead of using a $\xi-T_{\text {eff }}$ relation, using B as reference, excluding vanadium lines to derive effective temperature) does not change the abundance difference by more than 0.04 dex (peak to valley). As shown in Fig. 11, there are no trends in the abundance difference with $E W$, excitation potential and wavelength, supporting the reality of the observed difference.

The primary is most likely a blue straggler (Fig. 12). Considering the rarity of enriched pairs, it seems plausible that the abundance difference is in some way linked to the peculiar evolution of the primary. Possible scenarios are discussed in a separate paper (Desidera et al. 2006c).

- HIP 76603/2. The primary HIP $76603=$ HD 139461 is itself a spectroscopic binary (preliminary orbit by Tokovinin $\&$ Gorynya 2001; $P=888$ days, $e=0.9, K=$ $14 \mathrm{~km} \mathrm{~s}^{-1}$ ). The stars were also studied by Fuhrmann (2004). According to these studies, the Hipparcos distance is underestimated. Our analysis then used Fuhrmann spectroscopic distance. His spectroscopic analysis yields an abundance difference similar to ours (see Table 10). The spectroscopic
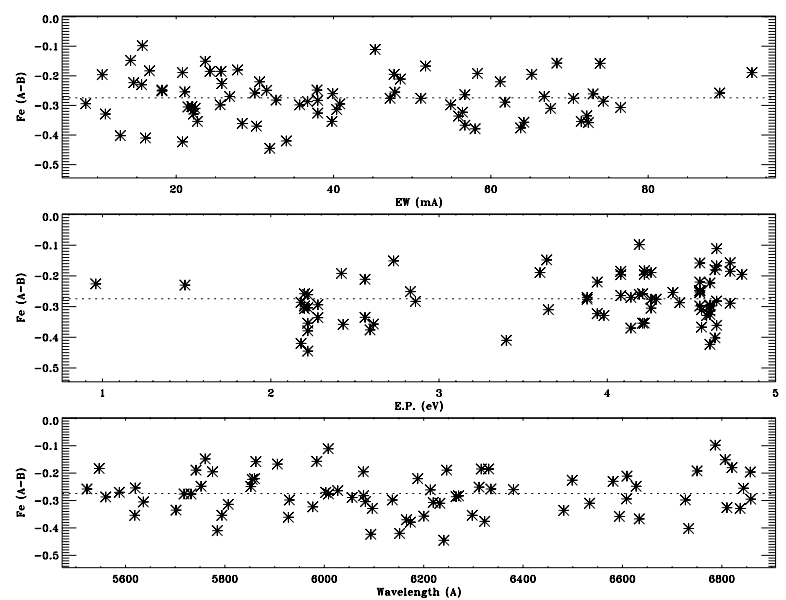

Fig. 11. Abundance difference of HIP 64030 as a function of $E W$ (upper panel), EP (middle panel) and wavelength (lower panel). No clear trends are present.

temperatures and gravities indicate that the two components should have very similar temperatures but slightly different gravities; i.e. the primary results evolved slightly (Fig. 3). This is compatible with the old age inferred from the low level of chromospheric activity (Paper II). On the other hand, the broad band colors support the presence of some temperature difference, with the primary being bluer (see Table 6). 

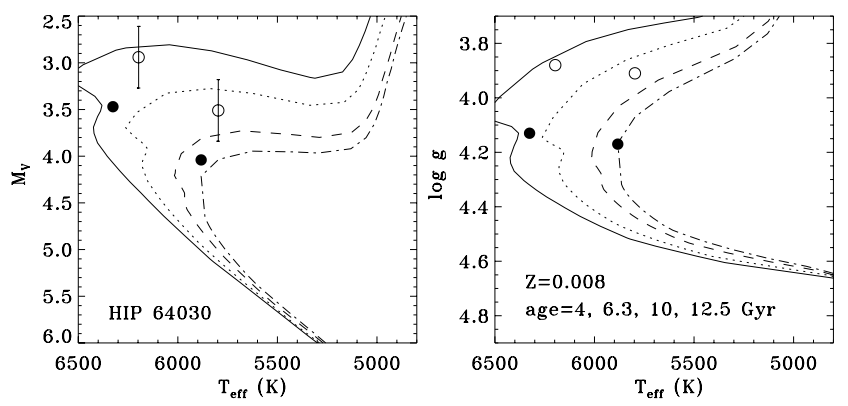

Fig. 12. Position of the components of HIP 64030 in the HR diagram (left panel) and in the $T_{\text {eff }}-\log g$ diagram (right panel). Open circles: results of abundance analysis performed when adopting the Hipparcos distance; filled circles: when adopting spectroscopic distances. Isochrones by Girardi et al. (2002) are overplotted.

However, Nordstrom et al. (2004) derived $\Delta T_{\text {eff }}=0$ and $\Delta[\mathrm{M} / \mathrm{H}]=-0.12$ from Strömgren photometry.

We investigated the possibility that the abundance difference is caused by the contamination of the spectrum of the primary by the light of its unseen companion. This should depend on the luminosity contrast and on the velocity difference. Fuhrmann (2004) derived a minimum mass for HIP $76603 \mathrm{~B}$ of about $0.34 M_{\odot}$. A companion contributing $5 \%$ and $1 \%$ of the light would have a mass of about 0.7 and $0.5 M_{\odot}$ respectively, corresponding to inclinations of 46 and $33 \mathrm{deg}$, respectively. The velocity difference for companions of such a mass with respect to that of HIP 76603A at the epoch of our spectrum as predicted by the Tokovinin \& Gorynya (2001) preliminary orbit is 5.6 and $7.0 \mathrm{~km} \mathrm{~s}^{-1}$.

The FWHM of the spectral lines of the primary measured on FEROS spectrum is about $12.2 \mathrm{~km} \mathrm{~s}^{-1}$. Therefore, the hypothetical spectral lines of HIP 76603B would be superimposed in a region of the line profile still within the region considered in our line-fitting procedure. The effects of such contamination on the measured equivalent widths should be small but possibly not negligible. On the other hand, the continuum contribution of HIP 76603B should make the measured $E W \mathrm{~s}$ smaller. In the case of contamination, we would expect a trend of abundance difference with wavelength, which is not observed (Fig. 13). Furthermore, asymmetries of the line profiles are seen neither by Fuhrmann nor in our spectrum. We plan to test the possibility of contamination further by observing this system at different phases of the spectroscopic orbit, then exploring the effect of the velocity difference between HIP 76603 A and B on the abundance difference ${ }^{3}$.

The alternative hypothesis is that the observed abundance difference is real and that the B component is enriched in heavy elements with respect to the primary. This would agree with the scenario devised by Laughlin (2000), which predicts that close pairs (spectroscopic binaries) should not be affected by pollution by planetesimal infall, while isolated stars (in this case the wide companion) might be.

Note that, while the mass of the companion would be compatible with that of a white dwarf, the very high orbital eccentricity $(e=0.9)$ makes a blue straggler scenario as for HIP 64030 unlikely, since these objects typically show low

${ }^{3}$ The epoch of the observation of Fuhrmann (2004) is not reported in his paper.

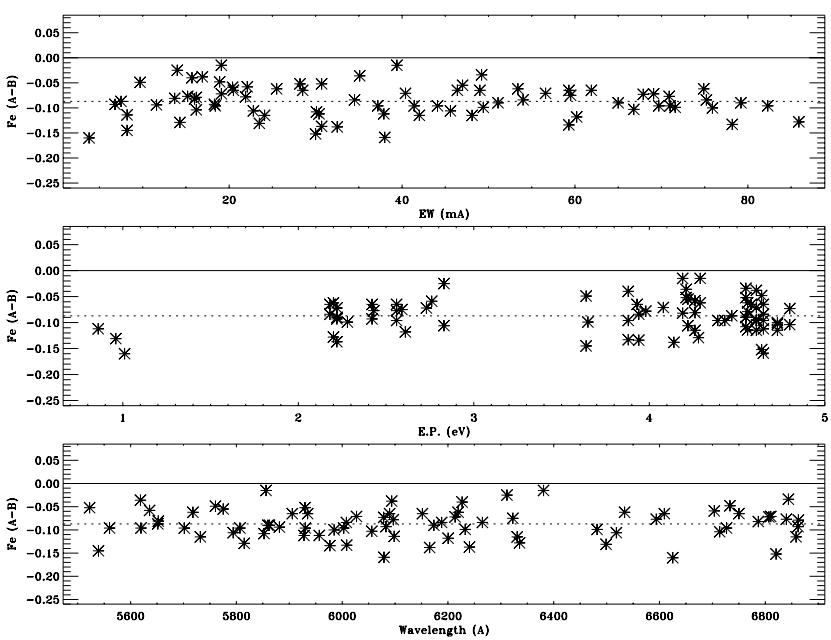

Fig. 13. Abundance difference of HIP $76602 / 3$ as a function of $E W$ (upper panel), EP (middle panel), and wavelength (lower panel). No clear trends are present.

eccentricities because of the circularization of the orbit during the mass transfer phase (Preston \& Sneden 2000).

- HIP 3290 and HIP 79818. These two pairs show a rather large abundance difference, with the primaries more metal poor than the secondaries. Their absolute abundances are sub-solar and the position in the color-magnitude diagram indicates a rather old age (about $6 \mathrm{Gyr}$ ) for both pairs, with significant evolution out of the main sequence. The analysis in both cases the analysis was performed by adopting the spectroscopic distance and by considering only spectral lines with equivalent width smaller than about $60 \mathrm{~m} \AA$. Nevertheless, some residual slope of abundance difference with equivalent width is still present.

A lower abundance for the relatively warm $\left(T_{\text {eff }} \sim\right.$ $6200-6100 \mathrm{~K}$ ) primaries of metal-poor pairs could be compatible with the diffusion of heavy elements, a scenario briefly discussed in Sect. 5.1.

Radial velocities of HIP 3290 from Paper II and Nordstrom et al. (2004) are constant within the errors. One of the components of HIP 79818 is instead a spectroscopic binary, since the radial velocity difference $\left(5.0 \mathrm{~km} \mathrm{~s}^{-1}\right)$ is not compatible with the binary motion of the wide pair (Paper II). This might explain the rather large error in parallax. We cannot exclude the occurrence of some contamination by this unseen companion.

- Active stars: HIP 37923/18, HIP 44817, HIP 70386. Five pairs show a rather high level of chromospheric activity ( $\left.\log R_{\mathrm{HK}}^{\prime} \geq-4.5\right)$ from our measurements (Paper II): HIP 37923/18, HIP 44817, HIP 51266, HIP 70386, and HIP 104687. Three of them (HIP 37923/18, HIP 44817, and HIP 70386) show abundance differences larger than 0.03 dex (formally significant at more than $2 \sigma$ level only for HIP 37923/18).

A strong magnetic activity and correlated phenomena may cause spurious abundance differences between the components of young, active pairs for several reasons:

- the adopted magnitude difference may be affected by photometric variability;

- the presence of spots may anomalously alter the strength of the vanadium lines (Dall et al. 2005), which are used to derive effective temperatures; 
- the atmospheric structure may have some systematic difference with respect to older inactive main sequence stars, introducing some effects on e.g. the adopted $T_{\text {eff-microturbulence relation (Padgett 1996); }}$

- differences in projected rotational velocity between the components (that are significant in our sample for HIP 37923/18 and HIP 70386) may cause spurious effects on $E W$ measurement by Gaussian fitting.

In the individual cases, HIP $37923 / 18$ is the most active pair in our sample $\left(\log R_{\mathrm{HK}}^{\prime}=-4.27\right.$ and -4.31 for $\mathrm{A}$ and $\mathrm{B}$ components respectively; Paper II) and has relatively different line widths of the two components $(v \sin i=6.0$ and $3.2 \mathrm{~km} \mathrm{~s}^{-1}$ ). When analyzed in a standard way, this pair shows a difference of 0.059 dex, but with a significant trend of abundance difference as a function of the $E W$. The analysis optimizing the values of the microturbulent velocity shows a smaller abundance difference but a large difference in the microturbulence, which is anomalous for stars with similar temperature.

- Pairs with a large temperature difference: HIP 76446, HIP 78024, HIP 103438. HIP 78024 and HIP 74436 have a magnitude difference above 1 mag and temperature differences above $700 \mathrm{~K}$. They were included in the sample because of the lack of suitable targets with a small magnitude difference observable at the end of the last night of the run. The abundance differences of -0.039 and -0.051 dex are compatible with the estimated errors and then are probably spurious. This confirms that the selection of pairs with similar components is mandatory to achieve a 0.02 dex error in the differential analysis.

Among the other pairs with large temperature difference, HIP 55288 and especially HIP 103438 are worth mentioning, because of the anomalous position in the HR diagram, with the secondary too bright for main sequence stars with the adopted temperature difference.

The discrepancy is fairly large for HIP 103438. The overluminosity of the secondary might be explained by binarity. The small radial velocity difference between the components found in Paper II argues against this hypothesis, but a singleepoch spectrum is not conclusive.

An alternative possibility for explaining the anomalous position in the color magnitude diagram would be a pre-main sequence status for the secondary. HIP 103438 was indeed considered for inclusion in the young Tucana-Horologium association but eventually discarded as a member, as it does not show the signatures of extreme youth expected for a 30 Myr age star (Zuckermann \& Webb 2000; Stelzer \& Neuhauser 2000; Paper II). In Paper II, we noted the rather large difference in the chromospheric activity level between the components. This may play a role in explaining the observed discrepancies. A full understanding of the properties of the system would require new high-precision photometric data, as the large difference between Hipparcos and Tycho magnitude differences is an indication of some problems in the measurement.

- HIP 20552, HIP 62596, HIP 73674. These are three pairs with small $(0.032-0.041 \mathrm{dex})$ but formally significant (at about $2 \sigma$ level) abundance difference. Two of them (HIP 62596, HIP 73674) have a very small temperature and magnitude difference. We did not identify specific problems in the analysis or in the stellar properties that may cause a spurious abundance difference. However, some of them might simply represent the tail of the Gaussian error distribution.
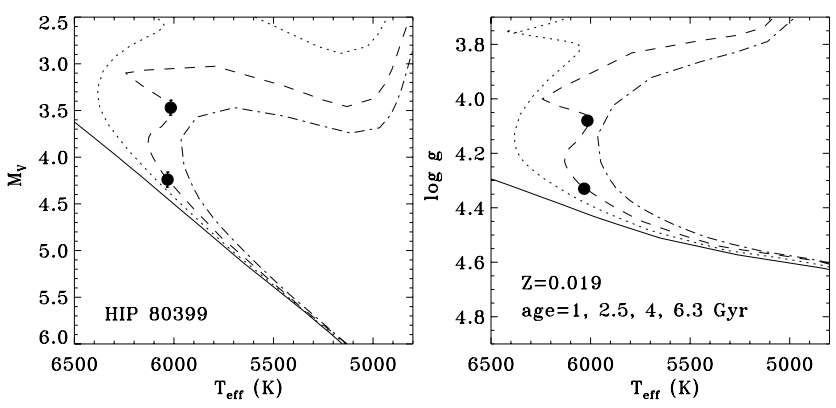

Fig. 14. Position of the components of HIP 80399 in the HR diagram (left panel) and in the $T_{\text {eff }}-\log g$ diagram, using absolute magnitude from Hipparcos parallaxes and effective temperature and gravities from our analysis. Overplotted are the 1, 2.5, 4.0 and $6.3 \mathrm{Gyr}$ isochrones of solar composition by Girardi et al. (2002).

\section{Discussion}

\subsection{Are we observing diffusion of heavy elements?}

Figure 8 shows that the scatter of abundance difference is larger for pairs with primaries warmer than $6000 \mathrm{~K}$. Furthermore, most of these pairs are fairly metal poor (Fig. 9), and the abundance difference is negative (smaller iron content of the primary than for the secondary). This might occur by chance or might be due to some unrecognized systematic effect in our analysis. Another intriguing possibility is that we are observing the signature of gravitational sedimentation of heavy elements, at least in some cases.

In the case of the Sun, the occurrence of diffusion is required to fit helioseismology data. From stellar models, we expect that the effects of diffusion should be stronger for stars with higher temperature (with higher mass and/or smaller metallicity); and then they might be observable as a metal deficiency of the primary in our differential analysis.

HIP 3290 and HIP 79818 are interesting candidates for diffusion, since they are old and moderately metal-poor, and have relatively warm primaries, and relatively large mass and temperature difference between the components. However, we note that HIP 58864 shares these properties, but its abundance difference is much smaller. The diffusion hypothesis is instead not plausible for HIP 76602/3, as the two components are very similar.

A quantitative comparison would require detailed theoretical predictions for the masses, ages, and metallicities of our pairs, which in general are not available. However, in the specific case of HIP 80399, we can use the theoretical predictions developed by Michaud et al. (2004) for the open cluster M 67, as their age and metallicity are similar (Fig. 14). The masses of HIP 80399 A and $\mathrm{B}$ as resulting from the $4 \mathrm{Gyr}$ isochrone in Fig. 14 are 1.24 and 1.09. The predicted iron abundance difference from Fig. 2 of Michaud et al. (2004) is about -0.03 and -0.02 dex for the models without and with turbulence ${ }^{4}$, respectively. The latter value is similar to the observed iron abundance difference of $-0.022 \mathrm{dex}$, but the error bar $(0.015$ dex $)$ does not allow us to claim that we detect heavy-element sedimentation with our data; however, we can reasonably conclude that the theoretical models considered here do not underestimate the diffusion of metals in stars that are slightly warmer than the Sun. A deeper investigation is postponed to a future paper.

${ }^{4}$ The parameterization of turbulence used in the quoted models was fixed to minimize the surface lithium abundance changes in Population II stars. 


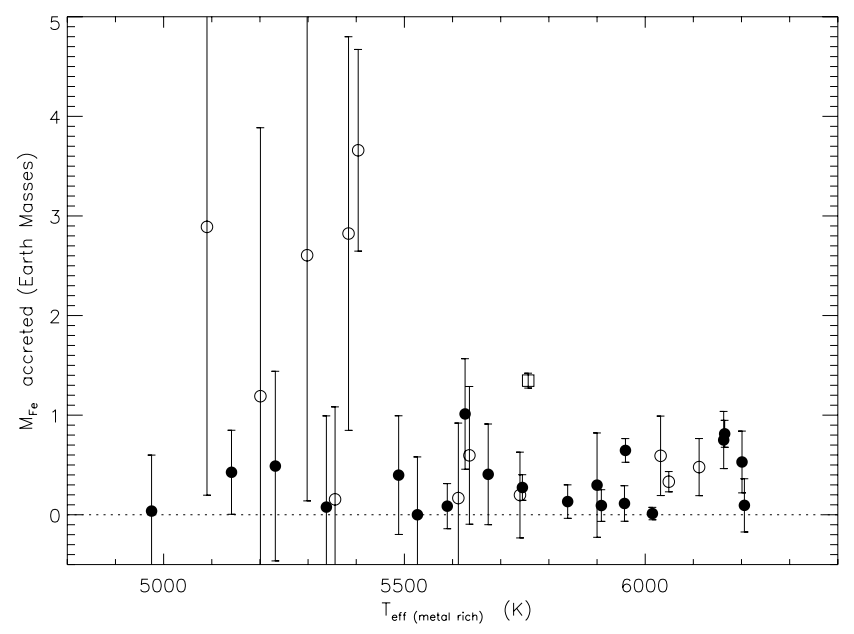

Fig. 15. Estimate of iron accreted by the metal-rich component of each pair as a function of its effective temperature, taking the mass of the mixing zone into account as in Murray et al. (2001). Symbols as in Fig. 7. The mass of meteoritic material is about 5.5 times the mass of iron.

\subsection{Clues to the accretion of metal rich material}

If we assume that the observed abundance differences are due to the accretion of metal rich material, we can estimate its amount by considering the actual stellar properties (extension of the convective zone, original metallicity). As in Paper I, we follow Murray et al. (2001) to estimate the extension of the mixing zone, which includes not only the standard convective zone but also the additional mixing zone required to explain the lithium dip.

Table 11 summarizes the results for abundance difference and shows the mass of iron accreted by the components that are more metal-rich. We note that, even for the pairs with relatively large abundance difference, the amount of rocky material required to explain the observed difference is rather small. In fact, most of these pairs are relatively warm and metal poor. For these stars it is much easier to have detectable abundance alteration, since the absolute metal content of the mixing zone is small $\left(\sim 3 M_{\oplus}\right.$ of iron for a star with $T_{\text {eff }}=6000 \mathrm{~K}$ and $[\mathrm{Fe} / \mathrm{H}]=-0.3)$. Therefore, if the differences are due to the ingestion of rocky material, the amount of accreted iron remains below $1 M_{\oplus}$ (except for HIP 64030).

After combining the results of this work with those of Paper I (Fig. 16), we found that for at least $65 \%$ of the pairs with temperature higher than $5500 \mathrm{~K}$, the estimated amount of iron accreted is smaller than that was expected to have been accreted by the Sun during the main sequence lifetime $\left(0.4 M_{\oplus}\right.$, Murray et al. 2001). Most of the pairs have a smaller abundance difference than the amount of iron corresponding to the upper limit on the abundance difference between the inner and outer regions of the Sun according to helioseismology $\left(2 M_{\oplus}\right.$, Winnick et al. 2002). These estimates are based on standard stellar models. If extramixing is induced by a metallicity enrichment of the convective envelope, as proposed by Vauclair (2004), a much larger amount of rocky material would be required to explain the observed abundance differences.

The frequency of pairs with large alteration of chemical composition appears to be small. If the abundance difference of HIP 64030 is indeed somewhat linked to the mass transfer event that lead to the formation of the blue straggler (as described in Desidera et al. 2006c), none of the 50 pairs

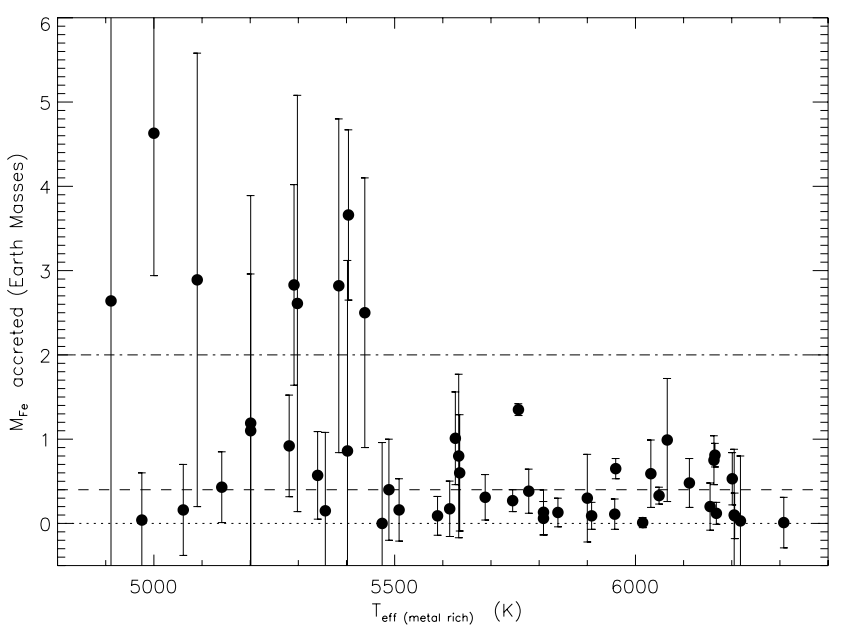

Fig. 16. Estimate of iron accreted by the metal-rich component of each pair as a function of its effective temperature for the pairs studied in this paper and in Paper I. The horizontal lines show the amount of iron expected to have been accreted by the Sun during the main sequence lifetime $\left(0.4 M_{\oplus}\right.$, Murray et al. 2001) and the amount of iron corresponding to the upper limit on abundance difference between the inner and outer regions of the Sun according to helioseismology $\left(2 M_{\oplus}\right.$, Winnick et al. 2002). The mass of meteoritic material is assumed to be about 5.5 times the mass of iron.

considered in this study and Paper I has an abundance difference larger than 0.1 dex. The few pairs with an abundance difference between 0.05 and 0.09 dex have mostly components warmer than $6000 \mathrm{~K}$ and with moderate metal deficiency. The amount of rocky material required to explain such abundance difference is then small.

Therefore, our study suggests that large alterations of chemical abundance caused by the ingestion of planetary material are rare and then cannot account for the strong correlation between the frequency of planets and metallicity. A possible caveat to a generalization of this conclusion is represented by the possibly lower frequency of planets in wide binaries with similar components. This is suggested by the lack of planet detection up to now in the radial velocity planet search we are performing using SARG at TNG (Desidera et al. 2006a).

Acknowledgements. This research made use of the SIMBAD database operated at the CDS, Strasbourg, France, and of data products from the Two Micron All Sky Survey, which is a joint project of the University of Massachusetts and the Infrared Processing and Analysis Center/California Institute of Technology, funded by the National Aeronautics and Space Administration and the National Science Foundation.

This work was partially funded by COFIN 2004 "From stars to planets: accretion, disk evolution and planet formation" by Ministero Università e Ricerca Scientifica Italy. We thank the referee Dr. N. Santos for his prompt report and stimulating comments.

\section{References}

Alonso, A., Arribas, S., \& Martinez-Roger, C. 1996, A\&A, 313, 873 Asplund, M. 2005, Ann. Rev. A\&A, 43, 481 Bessell, M. S. 2000, PASP, 112, 961

Bragaglia, A., Carretta, E., Gratton, R., et al. 2001, AJ, 121, 327

Bodaghee, A., Santos, N. C., Israelian, G., \& Mayor, M. 2003, A\&A, 404, 715

Cutri, R. M., Skrutskie, M. F., Van Dyk, S., et al. 2003, Explanatory Supplement to the 2MASS All Sky Data Release www.ipac. caltech. edu/2mass Cuypers, J., \& Seggewiss, W. 1999, A\&AS, 139, 425

Dall, T. H., Bruntt, H., \& Strassmeier, K. G. 2005, A\&A, 444, 573

Desidera, S., Gratton, R. G., Scuderi, S., et al. 2004, A\&A, 420, 683 (Paper I) 
Desidera, S., Gratton, R. G., Claudi, R. U., et al. 2006a, Proc. of the conference Tenth Anniversary of 51 Peg-b: status of and prospects for hot Jupiter studies, 119

Desidera, S., Gratton, R. G., Lucatello, S., Claudi, R. U., \& Dall, T. 2006b, A\&A, in press (Paper II)

Desidera, S., et al. 2006c, A\&A, in preparation

Docobo, J. A., Alvarez, C., Lahulla, J. F., Lancharesi, V., \& Aguirre, A. 2000, Astron. Nachr., 321, 53

ESA 1997, The Hipparcos and Tycho Catalogues, ESA SP-1200

Fischer, D., \& Valenti, J. 2005, ApJ, 622, 1102

Fuhrmann, K. 2004, Astron. Nachr., 325, 1

Girardi, L., Bertelli, G., Bressan, A., et al. 2002, A\&A, 391, 195

Gliese, W., \& Jahreiss, H. 1991, Preliminary Version of the Third Catalogue of Nearby Stars

Gonzalez, G. 1997, MNRAS, 285, 403

Gratton, R. G., Bonanno, G., Claudi, R. U., et al. 2001, A\&A, 377, 123

Kurucz, R. L. 1995, CD-ROM 13

Israelian, G., Santos, N. C., Mayor, M., \& Rebolo, R. 2003, A\&A, 405, 753

Laughlin, G. 2000, ApJ, 545, L1054
Laws, C., \& Gonzalez, G. 2001, ApJ, 533, 405

Magain, P. 1984, A\&A, 134, 189

Michaud, G., Richard, O., Richer, J., \& VandenBerg, D. A. 2004, ApJ, 606, 452

Murray, N., Chaboyer, B., Arras, P., Hansen, B., \& Noyes, R. W. 2001, ApJ, 555, 801

Nakos, T., Sinachopoulous, D., \& Van Dessel, E. 1995, A\&AS, 112, 453

Nissen, P. E., Hoeg, E., \& Schuster, W. J. 1997, Proc. of the ESA Symposium Hipparcos - Venice 97, ESA SP-402, 225

Nordstrom, B., Mayor, M., Andersen, J., et al. 2004, A\&A, 418, 989

Padgett, D. L. 1996, ApJ, 471, 847

Preston, G. W., \& Sneden, C. 2000, AJ, 120, 1014

Santos, N. C., Israelian, G., \& Mayor, M. 2004, A\&A, 415, 1153

Stelzer, B., \& Neuhauser, R. 2000, A\&A, 361, 581

Tokovinin, A. A., \& Gorynya, N. A. 2001, A\&A, 374, 227

Vauclair, S. 2004, ApJ, 605, 874

Winnick, R. A., Demarque, P., Basu, S., \& Guenther, D. B. 2002, ApJ, 576, 1075

Zuckerman, B., \& Webb, R. A. 2000, ApJ, 535, 959

Zuckerman, B., Song, I., \& Webb, R. A. 2001, ApJ, 559, 388 
S. Desidera et al.: Abundance difference between components of wide binaries. II., Online Material p 1

\section{Online Material}


S. Desidera et al.: Abundance difference between components of wide binaries. II., Online Material p 2

Table 2. Magnitude difference for program stars.

\begin{tabular}{|c|c|c|c|c|c|c|}
\hline$\overline{\overline{\text { Object }}}$ & $\begin{array}{c}\Delta V \\
\text { Hipparcos }\end{array}$ & $\begin{array}{c}\Delta V \\
\text { Tycho }\end{array}$ & $\begin{array}{c}\Delta V \\
\text { Other }\end{array}$ & $\begin{array}{c}\Delta V \\
\text { Other }\end{array}$ & $\begin{array}{l}\text { Ref. } \\
\text { Other }\end{array}$ & $\begin{array}{l}\Delta V \\
\text { Adopted }\end{array}$ \\
\hline HIP 3290 & $1.038 \pm 0.015$ & $0.917 \pm 0.021$ & $1.034 \pm 0.002$ & & 1 & $1.033 \pm 0.008$ \\
\hline HIP 10175 & $0.464 \pm 0.013$ & $0.477 \pm 0.034$ & $0.47 \pm 0.01$ & & 2 & $0.468 \pm 0.002$ \\
\hline HIP 20552 & $0.410 \pm 0.007$ & $0.390 \pm 0.015$ & & & & $0.406 \pm 0.008$ \\
\hline HIP 37923 & $0.106 \pm 0.024$ & $0.104 \pm 0.023$ & $0.104 \pm 0.009$ & $0.10 \pm 0.02$ & 3,4 & $0.104 \pm 0.001$ \\
\hline HIP 39409 & $0.057 \pm 0.013$ & $0.077 \pm 0.047$ & & & & $0.058 \pm 0.005$ \\
\hline HIP 44817 & $0.333 \pm 0.006$ & $0.273 \pm 0.045$ & $0.318 \pm 0.005$ & $0.296 \pm 0.009$ & 1,3 & $0.320 \pm 0.007$ \\
\hline HIP 49520 & $0.165 \pm 0.016$ & $0.158 \pm 0.033$ & $0.151 \pm 0.006$ & & 1 & $0.153 \pm 0.003$ \\
\hline HIP 51266 & $0.240 \pm 0.010$ & $0.232 \pm 0.023$ & & & & $0.239 \pm 0.003$ \\
\hline HIP 55288 & $0.753 \pm 0.016$ & $0.777 \pm 0.017$ & & & & $0.764 \pm 0.012$ \\
\hline HIP 56280 & $0.082 \pm 0.007$ & $0.108 \pm 0.006$ & $0.090 \pm 0.009$ & & 3 & $0.096 \pm 0.008$ \\
\hline HIP 58298 & $0.121 \pm 0.012$ & $0.059 \pm 0.035$ & & & & $0.114 \pm 0.019$ \\
\hline HIP 58813 & $0.687 \pm 0.049$ & $0.692 \pm 0.019$ & $0.686 \pm 0.009$ & $0.69 \pm 0.02$ & 3,4 & $0.687 \pm 0.001$ \\
\hline HIP 58864 & $0.673 \pm 0.018$ & $0.644 \pm 0.038$ & $0.64 \pm 0.02$ & & 4 & $0.657 \pm 0.011$ \\
\hline HIP 59743 & $0.273 \pm 0.009$ & $0.263 \pm 0.024$ & & & & $0.272 \pm 0.003$ \\
\hline HIP 62596 & $0.029 \pm 0.018$ & $-0.006 \pm 0.028$ & $0.065 \pm 0.009$ & $0.022 \pm 0.009$ & 1,3 & $0.040 \pm 0.013$ \\
\hline HIP 64030 & $0.566 \pm 0.009$ & $0.566 \pm 0.018$ & & & & $0.566 \pm 0.000$ \\
\hline HIP 65176 & $0.801 \pm 0.012$ & $0.722 \pm 0.021$ & $0.826 \pm 0.008$ & & 1 & $0.810 \pm 0.021$ \\
\hline HIP 65352 & $0.302 \pm 0.007$ & & $0.290 \pm 0.009$ & $0.30 \pm 0.02$ & 3,5 & $0.298 \pm 0.004$ \\
\hline HIP 69328 & $0.430 \pm 0.009$ & $0.380 \pm 0.025$ & $0.430 \pm 0.003$ & & 1 & $0.429 \pm 0.004$ \\
\hline HIP 70269 & $0.017 \pm 0.008$ & & & & & $0.017 \pm 0.008$ \\
\hline HIP 70386 & $0.175 \pm 0.011$ & $0.224 \pm 0.016$ & $0.238 \pm 0.009$ & & 3 & $0.215 \pm 0.020$ \\
\hline HIP 73674 & $0.016 \pm 0.019$ & $0.017 \pm 0.031$ & $0.008 \pm 0.009$ & & 3 & $0.010 \pm 0.003$ \\
\hline HIP 74432 & $0.894 \pm 0.020$ & $0.833 \pm 0.012$ & $0.833 \pm 0.009$ & $0.85 \pm 0.02$ & 3,4 & $0.841 \pm 0.011$ \\
\hline HIP 76446 & $1.231 \pm 0.019$ & $0.892 \pm 0.067$ & & & & $1.206 \pm 0.089$ \\
\hline HIP 76603 & $0.120 \pm 0.023$ & $0.078 \pm 0.013$ & $0.090 \pm 0.009$ & $0.02 \pm 0.02$ & 3,4 & $0.082 \pm 0.014$ \\
\hline HIP 78024 & $1.658 \pm 0.018$ & $1.601 \pm 0.028$ & & & & $1.641 \pm 0.026$ \\
\hline HIP 79818 & $0.611 \pm 0.014$ & $0.518 \pm 0.048$ & & & & $0.604 \pm 0.025$ \\
\hline HIP 80399 & $0.763 \pm 0.009$ & & & & & $0.763 \pm 0.009$ \\
\hline HIP 84405 & $-0.011 \pm 0.010$ & & $-0.04 \pm 0.02$ & & 5 & $-0.017 \pm 0.012$ \\
\hline HIP101292 & $0.020 \pm 0.009$ & & & & & $0.020 \pm 0.009$ \\
\hline HIP103438 & $0.599 \pm 0.012$ & $0.435 \pm 0.036$ & & & & $0.583 \pm 0.049$ \\
\hline HIP104687 & $0.048 \pm 0.008$ & $-0.052 \pm 0.053$ & $0.00 \pm 0.04$ & $0.06 \pm 0.04$ & 6,6 & $0.045 \pm 0.010$ \\
\hline HIP114914 & $0.369 \pm 0.010$ & $0.410 \pm 0.034$ & $0.391 \pm 0.007$ & & 1 & $0.384 \pm 0.008$ \\
\hline
\end{tabular}

References: 1: Cuypers \& Seggewiss (1999); 2: Nakos et al. (1995); 3: Nordstrom et al. (2004); 4: Simbad; 5: Gliese \& Jahreiss (1991); 6: Docobo et al. (2000). 
S. Desidera et al.: Abundance difference between components of wide binaries. II., Online Material p 3

Table 5. Results of abundance analysis for primaries.

\begin{tabular}{|c|c|c|c|c|c|c|c|c|c|}
\hline Object & {$[\mathrm{Fe} / \mathrm{H}] \mathrm{I}$} & $\mathrm{rms}$ & $N_{\text {lines }}$ & {$[\mathrm{Fe} / \mathrm{H}] \mathrm{II}$} & rms & $N_{\text {lines }}$ & {$[\mathrm{V} / \mathrm{H}] \mathrm{I}$} & $\mathrm{rms}$ & $N_{\text {lines }}$ \\
\hline HIP 3290 & 7.104 & 0.133 & 140 & 7.065 & 0.106 & 34 & 3.573 & 0.226 & 18 \\
\hline HIP 10175 & 7.440 & 0.095 & 137 & 7.340 & 0.187 & 39 & 3.778 & 0.117 & 33 \\
\hline HIP 20552 & 7.538 & 0.096 & 150 & 7.430 & 0.125 & 38 & 3.843 & 0.113 & 27 \\
\hline HIP 37923 & 7.704 & 0.126 & 69 & 7.678 & 0.215 & 30 & 4.192 & 0.223 & 35 \\
\hline HIP 39409 & 7.538 & 0.093 & 144 & 7.416 & 0.143 & 37 & 3.826 & 0.129 & 31 \\
\hline HIP 44817 & 7.741 & 0.105 & 66 & 7.614 & 0.206 & 33 & 4.227 & 0.124 & 30 \\
\hline HIP 49520 & 7.292 & 0.084 & 156 & 7.181 & 0.115 & 37 & 3.565 & 0.123 & 22 \\
\hline HIP 51266 & 7.528 & 0.118 & 121 & 7.504 & 0.128 & 28 & 4.021 & 0.197 & 34 \\
\hline HIP 55288 & 7.583 & 0.144 & 130 & 7.542 & 0.129 & 31 & 4.036 & 0.166 & 27 \\
\hline HIP 56280 & 7.531 & 0.121 & 147 & 7.419 & 0.135 & 32 & 3.848 & 0.144 & 17 \\
\hline HIP 58298 & 6.963 & 0.114 & 143 & 6.910 & 0.145 & 38 & 3.382 & 0.161 & 13 \\
\hline HIP 58813 & 7.588 & 0.094 & 146 & 7.460 & 0.130 & 37 & 3.883 & 0.063 & 22 \\
\hline HIP 58864 & 7.158 & 0.123 & 157 & 7.112 & 0.149 & 39 & 3.598 & 0.179 & 17 \\
\hline HIP 59743 & 7.525 & 0.095 & 137 & 7.485 & 0.188 & 33 & 3.973 & 0.122 & 31 \\
\hline HIP 62596 & 7.566 & 0.107 & 143 & 7.486 & 0.128 & 32 & 3.900 & 0.193 & 23 \\
\hline HIP 64030 & 6.904 & 0.194 & 138 & 6.868 & 0.122 & 41 & 3.463 & 0.305 & 11 \\
\hline HIP 65176 & 7.622 & 0.091 & 146 & 7.528 & 0.131 & 35 & 3.918 & 0.180 & 25 \\
\hline HIP 65352 & 7.087 & 0.097 & 155 & 7.075 & 0.127 & 24 & 3.579 & 0.076 & 30 \\
\hline HIP 69328 & 7.215 & 0.103 & 157 & 7.097 & 0.149 & 36 & 3.492 & 0.132 & 22 \\
\hline HIP 70269 & 7.229 & 0.098 & 153 & 7.141 & 0.141 & 39 & 3.566 & 0.133 & 21 \\
\hline HIP 70386 & 7.485 & 0.106 & 142 & 7.387 & 0.178 & 34 & 3.801 & 0.156 & 18 \\
\hline HIP 73674 & 7.191 & 0.095 & 159 & 7.106 & 0.116 & 34 & 3.533 & 0.136 & 26 \\
\hline HIP 74432 & 7.546 & 0.096 & 148 & 7.484 & 0.139 & 36 & 3.948 & 0.151 & 33 \\
\hline HIP 76446 & 7.427 & 0.080 & 121 & 7.343 & 0.117 & 35 & 3.777 & 0.157 & 30 \\
\hline HIP 76602 & 7.271 & 0.126 & 160 & 7.205 & 0.121 & 37 & 3.642 & 0.193 & 19 \\
\hline HIP 78024 & 7.576 & 0.090 & 131 & 7.483 & 0.128 & 35 & 3.895 & 0.149 & 24 \\
\hline HIP 79818 & 7.081 & 0.122 & 139 & 7.033 & 0.121 & 37 & 3.541 & 0.213 & 11 \\
\hline HIP 80399 & 7.601 & 0.080 & 117 & 7.497 & 0.145 & 35 & 3.940 & 0.097 & 26 \\
\hline HIP 84405 & 7.195 & 0.082 & 131 & 7.163 & 0.218 & 28 & 3.651 & 0.136 & 35 \\
\hline HIP 101292 & 7.559 & 0.098 & 144 & 7.463 & 0.149 & 35 & 3.891 & 0.158 & 36 \\
\hline HIP 103438 & 7.519 & 0.104 & 111 & 7.426 & 0.134 & 32 & 3.870 & 0.146 & 29 \\
\hline HIP 104687 & 7.569 & 0.111 & 150 & 7.500 & 0.141 & 38 & 3.953 & 0.150 & 31 \\
\hline HIP 114914 & 7.577 & 0.100 & 151 & 7.465 & 0.139 & 39 & 3.893 & 0.085 & 26 \\
\hline
\end{tabular}


S. Desidera et al.: Abundance difference between components of wide binaries. II., Online Material p 4

Table 6. Temperature difference using different methods. The weighted average between the temperature difference based on the ionization equilibrium of iron and vanadium was adopted because of its higher accuracy.

\begin{tabular}{|c|c|c|c|c|c|c|c|c|c|c|c|}
\hline$\overline{\text { Object }}$ & $\begin{array}{r}\text { Ion. Eq. } \\
\text { mean }\end{array}$ & $\begin{array}{r}\text { Ion. Eq. } \\
\text { Fe }\end{array}$ & $\begin{array}{r}\text { Ion. Eq. } \\
\mathrm{V}\end{array}$ & "Exc. Eq. & $\overline{\overline{\Delta V}}$ & $\overline{B B-V}$ & $\overline{\overline{V-I}}$ & $\overline{\overline{b-y}}$ & $\overline{\bar{V} V-K}$ & 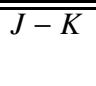 & $\overline{J-H}$ \\
\hline HIP 3290 & $143 \pm 11$ & $155 \pm 14$ & $127 \pm 16$ & $65 \pm 41$ & $\leq 577$ & 165 & 169 & & -31 & 65 & 191 \\
\hline HIP 10175 & $299 \pm 8$ & $264 \pm 13$ & $319 \pm 10$ & $238 \pm 21$ & $\leq 261$ & 186 & & & 176 & 487 & 273 \\
\hline HIP 20552 & $253 \pm 9$ & $210 \pm 14$ & $288 \pm 12$ & $214 \pm 16$ & $\leq 227$ & 250 & & & -98 & -388 & 252 \\
\hline HIP 37923 & $66 \pm 10$ & $63 \pm 15$ & $69 \pm 13$ & $17 \pm 49$ & $\leq 57$ & -16 & & 0 & 123 & 121 & 40 \\
\hline HIP 39409 & $25 \pm 11$ & $8 \pm 19$ & $34 \pm 14$ & $-1 \pm 18$ & $\leq 32$ & -252 & & & 97 & 434 & -416 \\
\hline HIP 44817 & $267 \pm 9$ & $243 \pm 14$ & $283 \pm 11$ & $271 \pm 47$ & $\leq 178$ & 43 & 140 & 118 & 180 & -10 & 169 \\
\hline HIP 49520 & $50 \pm 6$ & $43 \pm 8$ & $61 \pm 10$ & $30 \pm 14$ & $\leq 85$ & -64 & 136 & & 188 & 305 & 149 \\
\hline HIP 51266 & $148 \pm 10$ & $112 \pm 18$ & $166 \pm 12$ & $79 \pm 34$ & $\leq 133$ & -33 & & & 303 & 76 & 198 \\
\hline HIP 55288 & $579 \pm 10$ & $578 \pm 12$ & $583 \pm 20$ & $501 \pm 60$ & $\leq 427$ & 754 & & & 755 & 887 & 576 \\
\hline HIP 56280 & $73 \pm 10$ & $53 \pm 14$ & $95 \pm 15$ & $168 \pm 27$ & $\leq 53$ & -40 & & 71 & -133 & -95 & -88 \\
\hline HIP 58298 & $34 \pm 5$ & $36 \pm 5$ & $25 \pm 11$ & $63 \pm 12$ & $\leq 63$ & 144 & & & & & \\
\hline HIP 58813 & $376 \pm 9$ & $312 \pm 17$ & $400 \pm 10$ & $363 \pm 22$ & $\leq 384$ & 307 & 190 & 309 & -547 & -215 & 1605 \\
\hline HIP 58864 & $277 \pm 11$ & $280 \pm 13$ & $271 \pm 20$ & $274 \pm 25$ & $\leq 367$ & 677 & 701 & & 198 & 439 & 223 \\
\hline HIP 59743 & $164 \pm 9$ & $120 \pm 14$ & $195 \pm 11$ & $136 \pm 17$ & $\leq 151$ & 105 & & & 207 & 90 & 175 \\
\hline HIP 62596 & $-29 \pm 8$ & $-21 \pm 11$ & $-37 \pm 11$ & $-55 \pm 21$ & $\geq-22$ & -23 & -76 & -155 & -358 & -387 & -59 \\
\hline HIP 64030 & $439 \pm 14$ & $448 \pm 14$ & $323 \pm 53$ & $408 \pm 30$ & $\leq 316$ & 400 & & & 470 & 493 & 470 \\
\hline HIP 65176 & $391 \pm 8$ & $355 \pm 11$ & $426 \pm 11$ & $414 \pm 25$ & $\leq 452$ & 515 & 432 & & 839 & 669 & 699 \\
\hline HIP 65352 & $202 \pm 8$ & $172 \pm 14$ & $216 \pm 10$ & $237 \pm 16$ & $\leq 166$ & & 106 & 131 & 104 & 117 & -1 \\
\hline HIP 69328 & $163 \pm 8$ & $161 \pm 10$ & $166 \pm 15$ & $149 \pm 22$ & $\leq 240$ & 224 & 170 & & 255 & 18 & 214 \\
\hline HIP 70269 & $4 \pm 7$ & $12 \pm 8$ & $-20 \pm 15$ & $9 \pm 14$ & $\leq 9$ & & & & -69 & -167 & -121 \\
\hline HIP 70386 & $193 \pm 18$ & $202 \pm 23$ & $181 \pm 28$ & $324 \pm 32$ & $\leq 119$ & 250 & & 40 & 170 & 309 & 76 \\
\hline HIP 73674 & $5 \pm 7$ & $10 \pm 11$ & $0 \pm 10$ & $20 \pm 12$ & $\leq 5$ & -8 & & 26 & 10 & -146 & 32 \\
\hline HIP 74432 & $167 \pm 8$ & $130 \pm 13$ & $190 \pm 10$ & $61 \pm 24$ & $\leq 4 \overline{70}$ & 185 & 152 & 163 & 124 & -23 & 134 \\
\hline HIP 76446 & $720 \pm 18$ & $631 \pm 31$ & $771 \pm 23$ & $638 \pm 60$ & $\leq 674$ & 812 & & & 651 & 883 & 575 \\
\hline HIP 76602 & $-3 \pm 8$ & $1 \pm 9$ & $-19 \pm 18$ & $-19 \pm 18$ & $\leq 45$ & 131 & 40 & 0 & 230 & 158 & 428 \\
\hline HIP 78024 & $855 \pm 24$ & $794 \pm 33$ & $918 \pm 34$ & $594 \pm 50$ & $\leq 917$ & 902 & & & 571 & 368 & 946 \\
\hline HIP 79818 & $223 \pm 7$ & $226 \pm 7$ & $189 \pm 29$ & $184 \pm 26$ & $\leq 337$ & 377 & & & 180 & 135 & 447 \\
\hline HIP 80399 & $-16 \pm 4$ & $-16 \pm 8$ & $-16 \pm 5$ & $-32 \pm 17$ & $\leq 426$ & & & & & & 419 \\
\hline HIP 84405 & $-4 \pm 5$ & $-9 \pm 10$ & $-3 \pm 6$ & $25 \pm 10$ & $\leq-9$ & & & & & & \\
\hline HIP 101292 & $-8 \pm 5$ & $-13 \pm 9$ & $-6 \pm 6$ & $1 \pm 12$ & $\leq 11$ & & & & -114 & -72 & -13 \\
\hline HIP 103438 & $639 \pm 21$ & $515 \pm 36$ & $704 \pm 26$ & $490 \pm 62$ & $\leq 325$ & 526 & 547 & & 598 & 398 & 706 \\
\hline HIP 104687 & $40 \pm 5$ & $40 \pm 7$ & $41 \pm 8$ & $32 \pm 16$ & $\leq 24$ & 254 & & & -162 & 242 & 5 \\
\hline HIP 114914 & $151 \pm 7$ & $127 \pm 10$ & $175 \pm 10$ & $117 \pm 15$ & $\leq 214$ & 242 & 224 & & 23 & 219 & 232 \\
\hline
\end{tabular}


S. Desidera et al.: Abundance difference between components of wide binaries. II., Online Material p 5

Table 8. Sensitivity to abundance and temperature difference when changing of atmospheric parameters for a few selected pairs.

\begin{tabular}{|c|c|c|c|c|c|c|}
\hline Changes & $\Delta \mathrm{Fe} \mathrm{I}$ & $\Delta \mathrm{Fe}$ II & $\Delta \mathrm{VI}$ & $\begin{array}{c}\Delta T_{\text {eff }} \\
(\mathrm{Fe})\end{array}$ & $\begin{array}{c}\Delta T_{\text {eff }} \\
(\mathrm{V})\end{array}$ & $\begin{array}{l}\Delta T_{\text {eff }} \\
\text { mean }\end{array}$ \\
\hline \multicolumn{7}{|l|}{ HIP 114914} \\
\hline Reference & -0.013 & -0.033 & -0.068 & 21 & -23 & -1 \\
\hline$\Delta T_{\text {eff }}+30 \mathrm{~K}$ & -0.029 & -0.022 & -0.107 & -8 & -56 & -31 \\
\hline$\Delta T_{\text {eff }}-30 \mathrm{~K}$ & +0.008 & -0.044 & -0.034 & +57 & +6 & 31 \\
\hline$\Delta \log g+0.05 \mathrm{dex}$ & -0.009 & -0.053 & -0.067 & +49 & -9 & 21 \\
\hline$\Delta \log g-0.05 \mathrm{dex}$ & -0.015 & -0.012 & -0.068 & -2 & -37 & -20 \\
\hline$\Delta[\mathrm{A} / \mathrm{H}]+0.05 \operatorname{dex}$ & -0.017 & -0.048 & -0.068 & 35 & +9 & 12 \\
\hline$\Delta[\mathrm{A} / \mathrm{H}]-0.05 \mathrm{dex}$ & -0.007 & -0.018 & -0.067 & 11 & -32 & -10 \\
\hline$\Delta \xi+0.20 \mathrm{~km} \mathrm{~s}^{-1}$ & +0.019 & +0.013 & -0.051 & 6 & -42 & -17 \\
\hline$\Delta \xi-0.20 \mathrm{~km} \mathrm{~s}^{-1}$ & -0.041 & -0.074 & -0.091 & +36 & -11 & 11 \\
\hline \multicolumn{7}{|l|}{ HIP 73674} \\
\hline Reference & 0.033 & 0.037 & 0.045 & -4 & 5 & 0 \\
\hline$\Delta T_{\text {eff }}+30 \mathrm{~K}$ & 0.011 & 0.043 & 0.013 & -36 & -20 & -27 \\
\hline$\Delta T_{\text {eff }}-30 \mathrm{~K}$ & +0.053 & 0.029 & 0.077 & +26 & +31 & 29 \\
\hline$\Delta \log g+0.05 \mathrm{dex}$ & +0.034 & 0.017 & 0.045 & +19 & 18 & 19 \\
\hline$\Delta \log g-0.05 \mathrm{dex}$ & 0.028 & 0.057 & 0.045 & -32 & -8 & -18 \\
\hline$\Delta[\mathrm{A} / \mathrm{H}]+0.05 \mathrm{dex}$ & 0.030 & 0.025 & 0.044 & 5 & +12 & 9 \\
\hline$\Delta[\mathrm{A} / \mathrm{H}]-0.05 \operatorname{dex}$ & 0.034 & 0.049 & 0.045 & -15 & -2 & -8 \\
\hline$\Delta \xi+0.20 \mathrm{~km} \mathrm{~s}^{-1}$ & 0.052 & +0.066 & 0.074 & -15 & 5 & -6 \\
\hline$\Delta \xi-0.20 \mathrm{~km} \mathrm{~s}^{-1}$ & 0.008 & 0.009 & 0.050 & -1 & 27 & 14 \\
\hline \multicolumn{7}{|l|}{ HIP 84405} \\
\hline Reference & 0.001 & -0.004 & -0.005 & 5 & 0 & 0 \\
\hline$\Delta T_{\text {eff }}+30 \mathrm{~K}$ & -0.011 & 0.022 & -0.045 & -36 & -44 & -42 \\
\hline$\Delta T_{\text {eff }}-30 \mathrm{~K}$ & +0.011 & -0.026 & 0.032 & +41 & +38 & 39 \\
\hline$\Delta \log g+0.06 \mathrm{dex}$ & +0.003 & -0.023 & -0.002 & +28 & +14 & 18 \\
\hline$\Delta \log g-0.05 \mathrm{dex}$ & -0.001 & 0.017 & -0.009 & -19 & -17 & -17 \\
\hline$\Delta[\mathrm{A} / \mathrm{H}]+0.05 \mathrm{dex}$ & -0.012 & -0.026 & -0.008 & 15 & 11 & 12 \\
\hline$\Delta[\mathrm{A} / \mathrm{H}]-0.06 \mathrm{dex}$ & 0.017 & 0.028 & -0.001 & -12 & -19 & -17 \\
\hline$\Delta \xi+0.20 \mathrm{~km} \mathrm{~s}^{-1}$ & 0.008 & 0.010 & 0.007 & -3 & -2 & -2 \\
\hline$\Delta \xi-0.12 \mathrm{~km} \mathrm{~s}^{-1}$ & 0.000 & -0.005 & -0.007 & 6 & -1 & 0 \\
\hline \multicolumn{7}{|l|}{ HIP 56280} \\
\hline Reference & -0.006 & -0.025 & -0.057 & 20 & -21 & 0 \\
\hline$\Delta T_{\text {eff }}+30 \mathrm{~K}$ & -0.025 & -0.022 & -0.085 & -4 & -42 & -22 \\
\hline$\Delta T_{\text {eff }}-30 \mathrm{~K}$ & +0.013 & -0.030 & -0.029 & +47 & 0 & 24 \\
\hline$\Delta \log g+0.05 \mathrm{dex}$ & -0.005 & -0.045 & -0.057 & +44 & -7 & 19 \\
\hline$\Delta \log g-0.05 \mathrm{dex}$ & -0.008 & -0.006 & -0.057 & -2 & -34 & -17 \\
\hline$\Delta[\mathrm{A} / \mathrm{H}]+0.05 \operatorname{dex}$ & -0.008 & -0.035 & -0.059 & 30 & -16 & 8 \\
\hline$\Delta[\mathrm{A} / \mathrm{H}]-0.05 \mathrm{dex}$ & -0.006 & -0.018 & -0.056 & 13 & -25 & -4 \\
\hline$\Delta \xi+0.20 \mathrm{~km} \mathrm{~s}^{-1}$ & 0.023 & 0.013 & -0.050 & 10 & -42 & -15 \\
\hline$\Delta \xi-0.20 \mathrm{~km} \mathrm{~s}^{-1}$ & -0.034 & -0.071 & -0.065 & 41 & 3 & 21 \\
\hline
\end{tabular}

Table 9. Mean slopes of the variations in abundance difference and temperature difference between the components due to changes in the atmospheric parameters of the secondary.

\begin{tabular}{lccc}
\hline \hline Parameter & Change & $\Delta(\Delta[\mathrm{Fe} / \mathrm{H}](A-B))$ & $\Delta\left(\Delta T_{\mathrm{eff}}(A-B)\right)$ \\
\hline$\Delta T_{\mathrm{eff}}$ & $10 \mathrm{~K}$ & $0.0058 \pm 0.0008$ & $10.2 \pm 1.4$ \\
$\Delta \log g$ & $0.01 \mathrm{dex}$ & $0.0005 \pm 0.0002$ & $3.64 \pm 0.22$ \\
$\Delta[\mathrm{A} / \mathrm{H}]$ & $0.01 \mathrm{dex}$ & $0.0011 \pm 0.0006$ & $1.93 \pm 0.36$ \\
$\Delta \xi$ & $0.1 \mathrm{~km} \mathrm{~s}^{-1}$ & $0.0107 \pm 0.0033$ & $5.41 \pm 2.07$ \\
\hline
\end{tabular}

Table 10. Comparison of our analysis of HIP $76603 / 2$ with that by Fuhrmann (2004).

\begin{tabular}{lrrrr}
\hline \hline & $T_{\text {eff }}$ & $\log g$ & {$[\mathrm{Fe} / \mathrm{H}]$} & $\xi$ \\
\hline HIP 76603: US & 6162 & 4.27 & -0.25 & 1.57 \\
HIP 76603: F04 & 6250 & 4.26 & -0.18 & 1.17 \\
HIP 76602: US & 6165 & 4.31 & -0.16 & 1.57 \\
HIP 76602: F04 & 6236 & 4.32 & -0.12 & 1.16 \\
$\Delta(A-B):$ US & -3 & -0.04 & -0.09 & 0.00 \\
$\Delta(A-B):$ F04 & +14 & -0.06 & -0.06 & +0.01 \\
\hline
\end{tabular}

